\title{
Positionen im Umgang mit der historischen
}

Stadt

Hinsichtlich der Erhaltung des historischen Wien vertraten die städtische Verwaltung und die Denkmalschutzbehörde weitgehend unterschiedliche Auffassungen, woraus sich gegen Ende des Jahres 1937 ein ernsthafter Konflikt entwickelte. Wie es dazu kam, welche Standpunkte vertreten wurden und welche Rolle die institutionelle Denkmalpflege im Rahmen der Stadterneuerung einnahm, soll in diesem Kapitel hinterfragt werden.

\subsection{Denkmalpflege und Stadtbildkorrektur}

Mitte der 1930er-Jahre wurden in Wien, unabhängig von Assanierungs- und Hausreparaturfonds, auch andere „Stadtbildkorrekturen“ durchgeführt, die auch die institutionelle Denkmalpflege - im Einklang mit den Vorstellungen der Heimatschutzbewegung - sehr begrüßte. Kritik übte man vor allem am aufdringlichen Schmuck historistischer Fassaden. In diesem Sinne entfernte man etwa 1936, dank des „günstigen Zusammenwirkens“1 von Bauherr, Architekt, Stadtbauamt und Denkmalpflege, die pompöse Eckkuppel des Palais Herberstein am Michaelerplatz und rechtfertigte dies mit der nun besser zur Geltung kommenden Wirkung der Hofburgkuppel (Abb. 55).2 Auch in den Medien wurde der Umbau als „kunstsinnige und kulturelle Tat“ gepriesen, da sich - im Sinne eines einheitlichen, städtebaulichen Gestaltungswil-
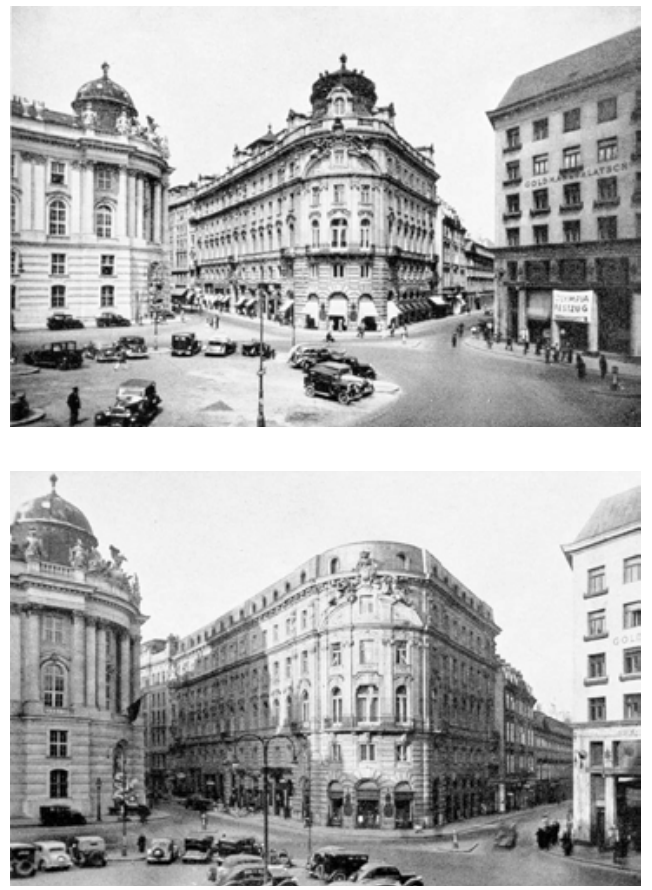

Abb. 55 Abtragung der Kuppel des Palais Herberstein am Michaelerplatz (Vorher-nachher-Vergleich), 1936 lens - alle umliegenden Gebäude dem Monumentalbau der Hofburg, als „künstlerischem Mittelpunkt“ des Michaelerplatzes, unterzuordnen hätten. ${ }^{3}$

Ebenso legitim waren für die Denkmalpflege ästhetisch motivierte „Korrekturen“ des Stadtbildes zur angeblichen Wiederherstellung einstiger städtebaulicher Gegebenheiten. 1937 wurde am Concordiaplatz ${ }^{4}$ ein ganzer Häuserblock abgetragen und ein neuer Stiegenaufgang zur Kirche Maria am Gestade errichtet, um „den Blick auf die reizvollste gotische Kirche Wiens frei zu bekommen“ (Abb. 56, 57). ${ }^{5}$ Begeisterte Stimmen waren zu hören: Aus „einem häßlichen Winkel der Stadt" befreit, blicke die Kirche nun „in ewig junger Schönheit [...] von ihrer Anhöhe herab, als wäre der Raum um sie von alters her so gewesen“.6 In der Österreichischen Kunsttopographie wurden die beiden abgetragenen Gebäude als ,an sich unbedeutend" und ihre plakatbedeckte Feuermauer gar als „abschreckend häßlich“ bezeichnet. Dennoch sollten bei einem „der Umgebung 


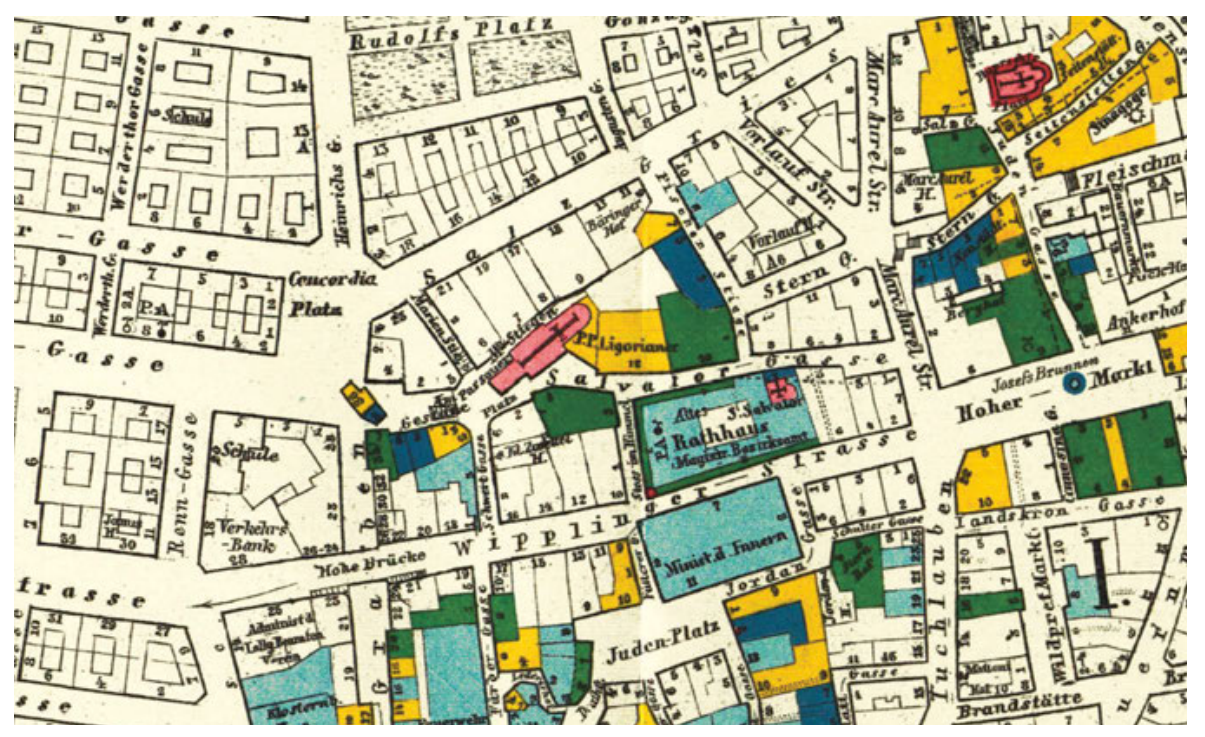

Abb. 56 Hugo Hassinger, „Kunsthistorischer Plan des 1. Bezirkes“, Ausschnitt, 1912; mit Einzeichnung der später abgebrochenen Häuser Tiefer Graben 36 (gelb) und Am Gestade 8 (blau) nahe der Kirche Maria am Gestade (Bildmitte)

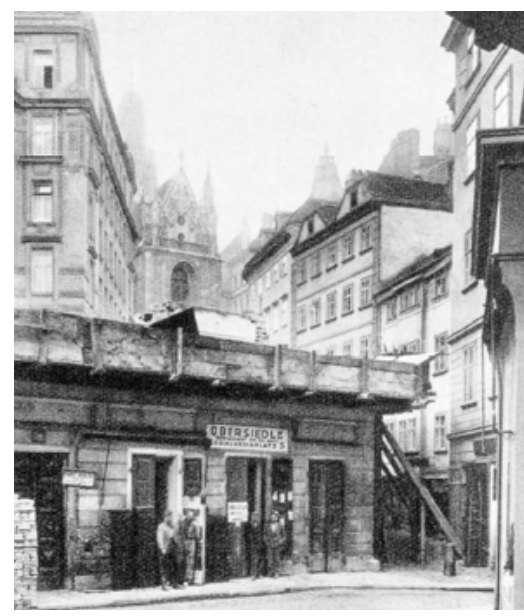

Abb. 57 Blick auf die Kirche Maria am Gestade während der Abbrucharbeiten und der Umgestaltung des Geländes vor der Kirche, Aufnahme vermutlich 1937

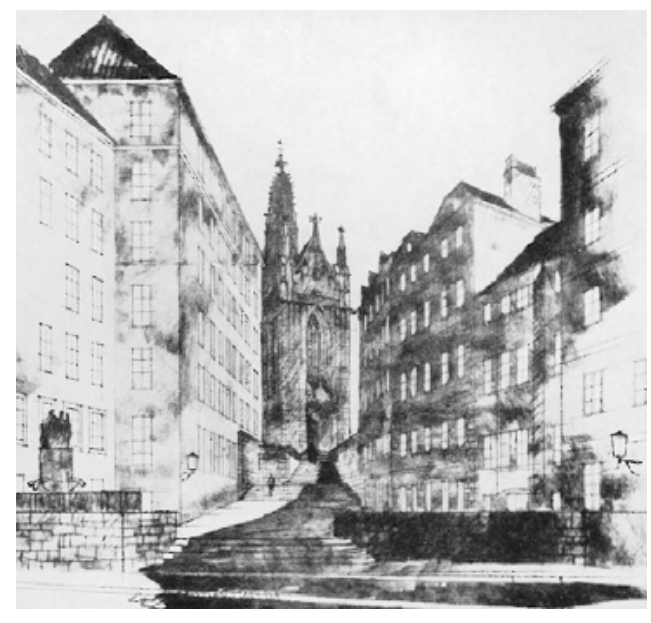

Abb. 58 Franz Hubert Matuschek, preisgekrönter Entwurf zur Neugestaltung der Stiegenanlage, 1936

entsprechenden Umbau“ die Gebäude und damit der Engpass „mit seinem schönen Durchblick auf die Kirche“ erhalten werden. ${ }^{\boldsymbol{7}}$ Dieser fortschrittlichen Auffassung von 1916, die sich im Sinne einer „modernen“ Denkmalpflege von einer Freistellung der Kirche in der Tradition des 19. Jahrhunderts klar distanzierte, wurde man 20 Jahre später schließlich nicht gerecht. Die damalige institutionelle Denkmalpflege zeigte sich erfreut über die Freilegung des Blicks auf die Kirche, die nun „wie ehemals“ wieder in ihrer reizvollen Umgebung zu sehen sei (Abb. 58). ${ }^{\mathbf{8}}$ Referent Karl Ginhart schlug sogar vor, auch noch zwei weitere Objekte an der Nordwand des Platzes, „zwei protzig hohe, 

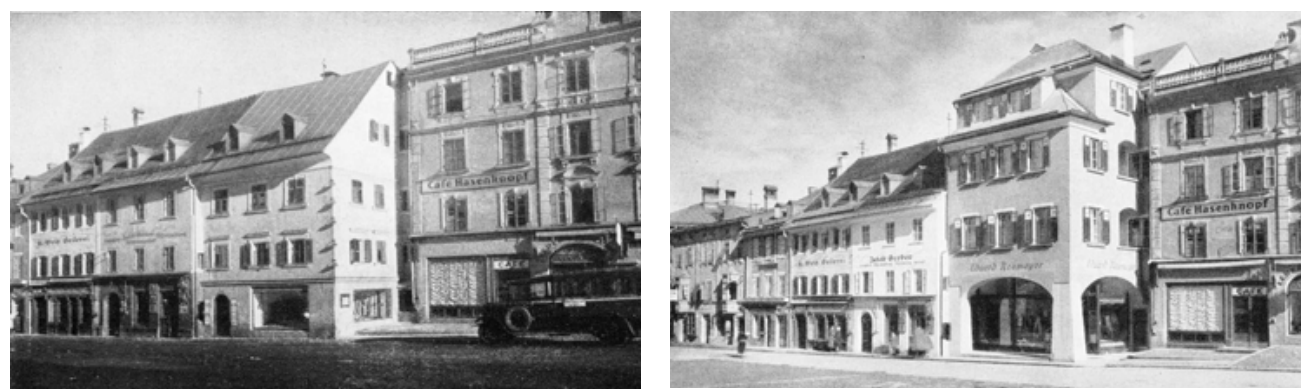

Abb.59 Eine Laube in Kufstein vor und nach der Realisierung, vor 1933

mit kleinlichen sezessionistischen Ornamenten sinnlos überladene Häuser von 1901, [...] abschreckende Beispiele aus einer bösen Zeit“9 (Am Gestade 2-4), zu entfernen, um eine endgültig befriedigende städtebauliche Lösung zu erzielen.

Wie diese Beispiele zeigen, befürworteten sowohl das Stadtbauamt als auch die Denkmalpflege in einzelnen Fällen die Beseitigung angeblicher städtebaulicher Irrtümer der Vergangenheit. Trotz der Befürwortung dieser „Korrekturen“ versuchte die Denkmalpflege dennoch, grundsätzlich den Verlust historischer Bausubstanz zu verhindern. Straßenverbreiterungen, die oftmals an beiden Straßenseiten den Abbruch von Altbauten erforderten, sollten vermieden werden. Um ganze Straßenzüge oder einzelne, in den Straßenraum hineinragende Gebäude zu erhalten, schlugen Städtebauer und Denkmalpfleger seit den 1920er-Jahren die Anlage von Fußgängerpassagen oder die Öffnung des Erdgeschosses durch Arkadengänge vor (Abb. 59).. ${ }^{10}$ Derartige Eingriffe findet man in Wien auch schon vor 1934: Bereits zwischen 1927 und 1929 wurden - nach Plänen von Carl Wilhelm Schmidt - Teile eines Nebengebäudes des Palais Schwarzenberg an der damaligen Heugasse (heute Prinz-Eugen-Straße) mit Arkaden geöffnet (Abb. 60)."1 Auch die ehemalige böhmische Hofkanzlei, ein Bau Johann Bernhard Fischer von Erlachs, sollte durch einen Fußgängerdurchgang vor der Zerstörung im Zuge einer geplanten Straßenverbreiterung bewahrt werden. Ab 1932 arbeitete man Pläne aus, ${ }^{12}$ eine Umsetzung fand das Projekt allerdings erst im Rahmen des Wiederaufbaus nach dem Zweiten Weltkrieg.

\subsection{Kompetenzfragen und Zuständigkeiten}

Gesetzliche Rahmenbedingungen für den Stadt- und Ortsbildschutz waren 1934 nur bedingt gegeben. In der damals gültigen Fassung der Bauordnung für Wien von 1929 sollte mittels des neu eingeführten $\S 87$ zur „Äußere[n] Gestaltung der Baulichkeiten“ gewährleistet werden, dass Neubauten hinsichtlich Bauform, Baustoff und Farbe „die einheitliche Gestaltung des örtlichen Stadtbildes" nicht störten. ${ }^{13}$ Darüber hinaus war es laut Bauordnung von nun an auch möglich, bauliche Änderungen an oder in der Umgebung von Gebäuden von ,geschichtlicher, kultureller und künstlerischer

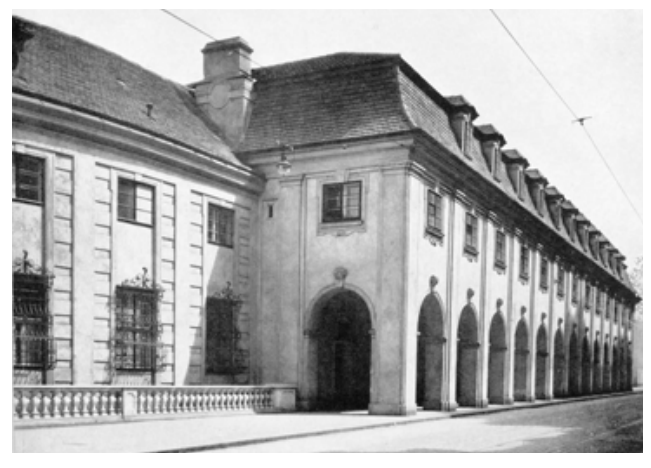

Abb. 60 Carl Wilhelm Schmidt, Nebentrakt des Palais Schwarzenberg an der Prinz-Eugen-Straße nach Anlage der Arkaden, 1927-1929 
Bedeutung“ zu untersagen, sofern „deren Eigenart oder künstlerische Wirkung“ dadurch beeinträchtigt werden würde. ${ }^{14}$ Wie die Beispiele zu Assanierungsbauten gezeigt haben, fügten sich diese grundsätzlich in die gegebene städtische Struktur ein. Die Gebäudehöhe folgte den Bestimmungen der Bauordnung und die Traufe glich sich somit den benachbarten Bauten zumeist an. Dennoch traten die Assanierungsbauten durch die hellfarbigen, glatt verputzten und nur sparsam oder gar nicht gegliederten Fassaden deutlich als Neubauten in Erscheinung.

Im Denkmalschutzgesetz von 1923 wird auf Aspekte des Ortsbildes nicht eingegangen, obwohl bereits Max Dvořák in seinem Katechismus der Denkmalpflege eindringlich auf die Bedeutung des Ensembles und die notwendige Erhaltung historisch gewachsener städtischer und dörflicher Strukturen hingewiesen hatte. Das Gesetz in der damaligen, ursprünglichen Fassung regelte nur auf das einzelne Objekt bezogene Fragen. ${ }^{15}$ Möglich war letztlich, parallel zu den zuvor zitierten Bestimmungen der Bauordnung, nur das Verbot von Veränderungen in der Umgebung von Denkmalen. ${ }^{\mathbf{1 6}}$ Dennoch fielen in den Wirkungsbereich der Zentralstelle auch Angelegenheiten der Stadt- bzw. Ortsbilderhaltung, da es sich dabei um eine Kernaufgabe der Heimatpflege handelte. ${ }^{17}$ Bereits 1923 war im damaligen Bundesdenkmalamt eine Fachstelle für Heimatschutz und Naturschutz unter der Leitung von Karl Giannoni eingerichtet worden, die auch nach der Neuorganisation im Jahr 1934 bestehen blieb. ${ }^{\mathbf{1 8}}$ Somit fiel im Sinne des öffentlichen Auftrags zur Heimatpflege der Orts- bzw. Stadtbildschutz auch in den Aufgabenbereich der Zentralstelle. Aus (bau-)rechtlicher Sicht lagen Aspekte der Altstadterhaltung und der Stadtregulierung im Zuständigkeitsbereich des Stadtbauamtes. Im Vorfeld der Einrichtung des Assanierungsfonds hatte man sich offenbar in einem Übereinkommen zwischen Stadtbauamt und Zentralstelle auf die jeweils bei der Denkmalschutzbehörde einzubringende Anfrage "um prinzipielle Stellungnahme“ geeinigt, sobald ein Förderansuchen zu einem Gebäude gestellt wurde.19

Mit der Einrichtung des Assanierungsfonds wurde die Zentralstelle für Denkmalschutz nochmals verstärkt in Fragen der Stadtregulierung involviert, was naturgemäß zu Unstimmigkeiten führte. Darüber hinaus waren durch das Bundesverfassungsgesetz von 1936 die Landeskonservatoren der Zentralstelle direkt dem jeweiligen Landeshauptmann unterstellt. ${ }^{20}$ Im Bundesland Wien war somit der oberste Denkmalschutzbeauftragte dem Bürgermeister der Stadt Wien untergeordnet, ${ }^{21}$ der, als Vorsitzender des fünfköpfigen Kuratoriums des Assanierungsfonds, in der Durchführung der Assanierung entscheidend mitwirkte. ${ }^{22}$

\subsection{Das Stadtbauamt - auf zu einer wirtschaftlich gesunden, modernen Stadt!}

„Solche Verkehrshindernisse sind in allen inneren Bezirken, teilweise aber auch in den äußeren Wiener Gemeindebezirken vorhanden. [...] Im allgemeinen wäre noch zu sagen, daß man bei der Beseitigung solcher Verkehrshindernisse, die häufig unter Denkmalschutz stehen, nicht allzu ängstlich sein dürfte. Wien hat so viele historische Baudenkmäler überragender Art, daß es auf die Entfernung des einen oder anderen älteren, wenn auch künstlerisch oder historisch interessanten Hauses nicht unbedingt ankommen darf, wenn die Modernisierung der Großstadt und die Verbesserung des Verkehrs es erfordern." ${ }^{23}$ 
Diese Auffassung entspricht jener der Stadtplaner und Stadterneuerer, wie sie sich in den entsprechenden Abteilungen des Wiener Magistrates wiederfanden. Die Regulierungsmaßnahmen verstand man hier als absolute Notwendigkeit, aus verkehrsplanerischer und nicht zuletzt auch aus ästhetischer Sicht. Mit der Regulierung und Assanierung und durch Großprojekte, wie dem Bau der Wiener Höhenstraße, beabsichtigte man eine „Verschönerung“ des Stadtbildes, die letztlich auch eine Belebung des Fremdenverkehrs bewirken sollte. ${ }^{\mathbf{2 4}}$ Hauptziel der Förderung der privaten Bauwirtschaft war es, die bestehende Arbeitslosigkeit zu senken, aber es stellte sich eben auch ein positiver „Nebeneffekt“ ein. 1935 stellte das Stadtbauamt in einer Rückschau auf sein 100-jähriges Bestehen erfreut fest, dass man nun endlich auch „schöpferisch tätig“ geworden sei und Einfluss auf die baukünstlerische Entwicklung Wiens genommen habe. ${ }^{\mathbf{2 5}}$ Neben der verkehrstechnischen Notwendigkeit der Regulierung rückten die offiziellen Meldungen aus dem Stadtbauamt nun auch die ästhetische Verbesserung in den Vordergrund, wie man im Falle der Begradigung der Wollzeile gegenüber der Zentralstelle festhält:

„Es ist sicher, dass [...] das heutige, hässliche Stadtbild der Wollzeile mit seinen vor-
und rückspringenden Häusern, seinen ungleichmässigen [sic!] Gehsteighöhen und
provisorischen Stiegenanlagen einen geradezu abstossenden [sic!] Eindruck her-
vorrufen wird; wohingegen eine ausregulierte Verkehrsstrasse [sic!] mit moder-
nen Geschäften den Eindruck einer wirtschaftlich gesunden Stadt hervorzurufen
geeignet ist.“26

Das Wiener Stadtbauamt nahm vor allem durch die Festlegung der neuen, zurückversetzten Baulinien auf die Stadtregulierung und auch auf Fragen des Stadtbildes Einfluss. Die streng gezogenen Linien machten erst die verkehrsbehindernde Lage der Altbauten deutlich und erzwangen geradezu deren Abriss.

Einen besonders radikalen Ansatz vertrat Stadtbaumeister Herbert Soche, der 1934 für eine Übernahme der Assanierungsangelegenheiten durch die öffentliche Hand plädierte und gar eine durch die Bezirksbauämter durchgeführte, planmäßige Assanierung forderte, die „alle den Verkehr hindernden Objekte, Engpässe und Gefahrenpunkte“ je nach Dringlichkeit schrittweise entfernen sollte. Durch eine derartige „Altstadtsanierung wäre nach einer gewissen Reihe von Jahren endlich die so dringend nötige und zur Lebensfrage gewordene bauliche Ordnung in die Stadt und das städtische Verkehrswesen gebracht“. ${ }^{27}$ Es sollte also vorrangig den Anforderungen des Verkehrs Rechnung getragen werden, angeblich „unter besonderer Berücksichtigung“ der Baudenkmäler. Diese Rücksichtnahme sah er allerdings schon durch die Wiederverwendung charakteristischer Teile der zerstörten Gebäude, wie Portale oder Fensterlaibungen, als gegeben. Grundsätzlich sollte bei Baudenkmalen „bei aller Pietät und Würdigung nicht gar zu ängstlich vorgegangen werden“.28 Eine planmäßige, von der Gemeinde durchgeführte Assanierung konnte die Stadt Wien aufgrund der rechtlichen und finanziellen Situation allerdings in den 1930er-Jahren nicht leisten.

Vor allem Stadtbaudirektor Franz Musil ${ }^{\mathbf{2 9}}$ befürwortete die Regulierung der Inneren Stadt. Die Proteste aus der Bevölkerung, die im Jänner 1938 deutlicher denn je wahrzunehmen waren, kamen seiner Ansicht nach „Von lebensfremden, einseitige ästhetische und kulturhistorische Interessen verfolgenden Kreisen“, denen „die Beschäftigung des Wiener Baugewerbes völlig fremd und gleichgültig“" sei. ${ }^{30}$ Die be- 
wusste Erhaltung bedeutender Altstadtteile vertrug sich seiner Ansicht nach nicht mehr „mit den Erfordernissen des intensiven Verkehres und Wirtschaftslebens einer Zweimillionenstadt“. ${ }^{31}$ Lediglich von allzu radikalen Vorschlägen, wie beispielsweise dem Straßendurchbruch vom Laurenzerberg zur Akademiestraße, die noch aus der Monarchie stammten, nahm er Abstand. ${ }^{32}$ Eine von der Architektenschaft geforderte generelle Überarbeitung der bestehenden Regulierungspläne lehnte er ab, da diese seiner Ansicht nach nur unnötige Kosten verursachen und kein „brauchbares Resultat" ergeben würde. ${ }^{33}$ Darüber hinaus befand er die laufende Anpassung der Pläne an die veränderten Bedingungen und Anforderungen durch das Stadtbauamt als ausreichend. ${ }^{34}$ Außerdem habe man mit der Novellierung der Bauordnung für Wien auch einen Fachbeirat für städtebauliche Fragen als zusätzliche Kontrollinstanz eingeführt, zusammengesetzt aus „unabhängigen und hervorragenden Fachleuten“.35

4.4 Die Zentralstelle - Hüterin der „wenigen letzten Zeugen der Wiener Vergangenheit“36 Entscheidende Auswirkungen hatten die politischen Veränderungen im Jahr 1934 auch auf die staatliche Denkmalpflege. Am 25. Mai 1934 wurde das Bundesdenkmalamt per Bundesgesetz aufgelassen und seine Agenden dem Bundesministerium für Unterricht übertragen. Denkmalschutz und Denkmalpflege waren von nun an Aufgaben der „Zentralstelle für Denkmalschutz“,37 einer dem Ministerium zugeordneten Dienststelle unter der Leitung von Leodegar Petrin ${ }^{38}$.

Agenden, die bisher im Zuständigkeitsbereich des Ministeriums lagen, wurden nun von der Zentralstelle übernommen, und auch Aufgaben des Denkmalschutzes, wie etwa Unterschutzstellungen oder die Vergabe von Förderungen, lagen bei der Zentrale in Wien. Zur Unterstützung der Denkmalbehörde konnten zusätzlich ehrenamtliche Landeskonservatoren für maximal fünf Jahre bestellt werden. Darüber hinaus war es fortan möglich, in den Bereichen Denkmal- und Heimatpflege fachlich versierte Personen ebenfalls für fünf Jahre als „Korrespondenten“ zu berufen. ${ }^{\mathbf{3 9}}$ Mit der Neufassung des Statuts von 1936 wurde darüber hinaus ein „Beirat für Fragen des Bauwesens auf dem Gebiet der Denkmalpflege“ als beratendes Organ geschaffen, der aus mindestens drei Fachleuten aus dem Bereich der Baukunst bestand, die jeweils für die Dauer eines Kalenderjahres berufen wurden..$^{\mathbf{4 0}}$ Der Beirat wurde von der Zentralstelle bei diversen Regulierungsfällen in beratender Funktion hinzugezogen und erteilte beispielsweise auch entscheidende Gestaltungsvorgaben für den Neubau des Dominikanerklosters in der Wollzeile (siehe dazu Kap. 5.1.4.).

Nach derzeitigem Forschungsstand schwand mit der Machtübernahme der christlich-autoritären Staatsführung der Einfluss der staatlichen Denkmalpflege auf die Umsetzung des Denkmalschutzes: Der Handlungsspielraum beschränkte sich auf administrative Tätigkeiten. ${ }^{41}$ Während Frodl-Kraft die Zeit zwischen 1934 und 1938 als „Jahre der Stagnation“42 bezeichnet, blieben, so der Zeitzeuge Otto Demus, die wesentlichen organisatorischen Strukturen „beim Alten“ und das vorhandene Fachpersonal wahrte „die Kontinuität der österreichischen Denkmalpflege“.43 Ernst Bacher hingegen spricht hinsichtlich der staatlichen Denkmalpflege dieser Jahre von einer reinen „Denkmalverwaltung“, in der die „Administration den fachlichen Bereich dominierte bzw. ersetzte“.44

Finanzielle und personelle Engpässe konnten dank der Unterstützung durch ehrenamtliche Korrespondenten und Konservatoren ausgeglichen werden, die der 
Zentrale zu laufenden Restaurierungen und Heimatschutzangelegenheiten Bericht erstatteten.45 Für eine effektive und weitgreifende Umsetzung des Denkmalschutzgesetzes fehlten laut Frodl-Kraft dem Amt aber nicht nur die finanziellen Mittel, sondern auch die moralische Unterstützung aus der Bevölkerung.46 Die finanzielle Lage war, wie auch bereits in den Jahren vor dem Ersten Weltkrieg, derart misslich, dass man nur wenige Restaurierungsarbeiten in Angriff nehmen konnte und sich stattdessen vor allem einer umfangreichen Publikationstätigkeit widmete und die wissenschaftliche Erfassung und Inventarisation des Denkmalbestandes vorantrieb. ${ }^{\mathbf{4 7}}$

1933 erfuhr der ohnehin knappe Personalstand eine weitere Kürzung. Alle juristischen Angelegenheiten mussten fortan von Präsident Leodegar Petrin selbst übernommen werden, dabei standen neben sechs wissenschaftlichen Mitarbeitern nur noch zwei „technische Beamte“ (Architekten) zur Verfügung.48 Auf den besorgniserregenden Personalstand verweist 1936 auch Karl Ginhart. Bei seiner Berufung an den Lehrstuhl für Kunstgeschichte an der Technischen Hochschule Wien bot er der Zentralstelle „angesichts des nahezu dezimierten Personalstandes“ an, für die Dauer von zwei Jahren weiterhin seine Agenden in der Zentralstelle - als Leiter des Kunsthistorischen Institutes - zu übernehmen. Anfang des Jahres 1936 waren dort nur noch vier Kunsthistoriker beschäftigt, weshalb man es auch als „absolute Notwendigkeit“ erachtete, „Von diesem Anbote Gebrauch zu machen“.49 Am Ende desselben Jahres wurde es aufgrund der „trostlosen Personalverhältnisse“ als unumgänglich befunden, mit Otto Demus, zum damaligen Zeitpunkt Landeskonservator von Kärnten, einen „erfahrenen jüngeren Beamten“ zum Dienst nach Wien einzuberufen. ${ }^{\mathbf{5 0}}$ Von den Investitionen in die Arbeitsbeschaffungsmaßnahmen zu profitieren, stellte somit auch für die Denkmalpflege eine berechtigte Hoffnung dar. Bereits im August 1933 hatte Präsident Leodegar Petrin die Landeskonservatorate über die geplante Maßnahme informiert, von nun an die ,junge, arbeitslose Künstlerschaft in den freiwilligen Arbeitsdienst (FAD) einzubeziehen" und hatte die dringende Veranlassung entsprechender Schritte gefordert.51

Die beschränkten Personalressourcen wirkten sich natürlich auch auf die Handlungsfähigkeit des Amtes aus. In den Archivakten der Topographischen Sammlung aus den Jahren 1934 bis 1938 scheint als zeichnender Referent fast ausschließlich Emmerich Siegris auf, der somit eine schier unglaubliche Anzahl an Objekten zu besichtigen und zu bewerten hatte. Nicht zuletzt aufgrund der Personalsituation stand man der Assanierung der 1930er-Jahre machtlos und scheinbar tatenlos gegenüber. Wesentlich für diese Lage waren jedoch die fehlenden - beziehungsweise nicht ausreichenden - Rechtsmittel, um den drohenden Abbruch vieler Objekte zu verhindern. 52 Galt ein Gebäude auf Basis der damals gültigen Kriterien als nicht schutzwürdig, so war die „gewiss bedauerliche“53 Demolierung nicht zu verhindern. Aus zahlreichen Kommentaren in den Akten des Bundesdenkmalamtes geht diese fehlende Handlungsmöglichkeit klar hervor. Viele Objekte wurden zwar für das Gesamtbild und Gefüge historischer Ensembles als durchaus „erhaltenswert“ eingestuft, ${ }^{\mathbf{4}}$ die im Denkmalschutzgesetz von 1923 festgehaltenen Kriterien der Schutzwürdigkeit bei „geschichtlicher, künstlerischer oder kultureller Bedeutung“ eines Gebäudes sah man jedoch letztlich als nicht erfüllt.

Auf Basis des Denkmalschutzgesetzes wurden in den Jahren nach 1923 im Bereich der unbeweglichen Denkmale neben Burganlagen, Schlössern, historischen Stadt- 
befestigungen und Ausgrabungsstätten nur die bedeutendsten privaten Wohnhäuser von hohem künstlerischen Wert unter Denkmalschutz gestellt, wie etwa das „Töpfelhaus" in Wien-Penzing, dessen Unterschutzstellung bereits 1928 erfolgte. ${ }^{55}$ Die in vielen Bereichen der Stadt noch durchaus zahlreich vorhandenen Bürgerhäuser des späten 18. und frühen 19. Jahrhunderts, die in großer Zahl der Assanierung der 1930er-Jahre zum Opfer fielen, wurden zum damaligen Zeitpunkt nur bei außerordentlicher künstlerischer Qualität für eine Unterschutzstellung berücksichtigt. In Kapitel 4.6. wird das Thema der denkmalfachlichen Bewertung noch ausführlicher behandelt werden.

Für die amtliche Denkmalpflege waren die vorhandenen Altstadtensembles, vor allem jene der Inneren Stadt, aufgrund ihres Stimmungswertes grundsätzlich erhaltenswert, wie den Aufzeichnungen der Referenten zu entnehmen ist. Für den behördlichen Schutz und die Erhaltung der charakteristischen Ortsbilder sah man sich weder zuständig, trotz der 1923 eingerichteten Fachstelle für Heimatschutz und Naturschutz, die sich gerade um Ortsbildfragen bemühte, noch befugt. ${ }^{56}$ Aufgrund der bestehenden Rechtslage verstand man es als alleinige Aufgabe des Wiener Stadtbauamtes, für die Wahrung des Stadtbildes Sorge zu tragen, die die Behörde allerdings nicht wahrnahm. ${ }^{\mathbf{5 7}}$

\subsection{Kontroversen um die Zukunft von „Alt-Wien“}

Die Zentralstelle habe „bei Umbauten in der Inneren Stadt wiederholt grosse [sic!] Schwierigkeiten bereitet“, hält Stadtbaudirektor Musil im November 1937 fest. Diese Aussage verdeutlicht das - bereits seit Jahrzehnten - zerrüttete Verhältnis zwischen Stadtverwaltung und institutioneller Denkmalpflege. ${ }^{\mathbf{5 8}}$ Die Regulierungsabsichten des Stadtbauamtes trafen in den Reihen der Zentralstelle für Denkmalschutz nur selten auf Verständnis. Vor allem am Beginn der Assanierungsaktion finden sich zwar noch mehrfach Anmerkungen, dass Gebäude aufgrund ihrer verkehrsbehindernden Lage nicht erhalten oder gar geschützt werden könnten ${ }^{\mathbf{5 9}}$ und die „Privatinteressen und die Interessen der Denkmalpflege [...] vor den öffentlichen Verkehrsinteressen [...] leider zurückstehen" müssten.60 Es zeigt sich aber ein gewisser Prozess in der Position der amtlichen Denkmalpflege, die mit fortschreitendem Verlust an Gebäuden auch die Situation zunehmend kritisch beurteilte.

Die kritischen Äußerungen reichen von der Feststellung der „Machtlosigkeit“61 gegenüber den Interessen der Gemeinde und der Verbreiterung zu enger Straßen bis hin zu klaren Schuldzuweisungen. Es wird etwa von der „geschickten Taktik der Gemeinde Wien" gesprochen, die Objekte gezielt aufkaufe, um sie zuerst verfallen und dann in weiterer Folge abreißen zu lassen.62 ${ }^{62}$ Objekte im Besitz der Stadt Wien standen oder in deren Besitz übergingen, sah man die Gefahr einer Veränderung offenbar als besonders gegeben. Die fehlenden Möglichkeiten, diesen Prozess zu unterbinden, schürten einen stetig wachsenden Unmut in den Reihen der Zentralstelle. Letztlich stellte sich auch ein gewisses Gefühl der Ohnmacht ein, da zumeist nichts anderes übrig blieb, „als noch Fotografien des Hauses zu verlangen und den Demolierungsauftrag zur Kenntnis zu nehmen“.63 Mit fortdauernder Regulierungstätigkeit war man zunehmend auch um die Wahrnehmung und das „Ansehen der Denkmalbehörde“ besorgt. ${ }^{64} \mathrm{Trotz}$ der Meinungsverschiedenheiten hoffte man, in den Reihen des Stadtbauamtes durch Aufklärung größeres Verständnis für denkmalpflegerische Anliegen zu erlangen. 1937 wandte man sich im Zusammenhang mit dem geplanten Abriss des Palais Paar mit gewichtigen und mahnenden Worten an die Stadt Wien: 
„Heute, da auf der ganzen Welt solche historische Stätten dem modernen Großstadtmenschen einen letzten Rest von Heimat und Bodenverbundenheit geben und daher fast in allen Großstädten Europas [...] nicht nur aus ästhetischen, sondern auch aus praktisch-volkspolitischen Gründen geschützt werden, fühlt sich die Z.St. f. D. [Zentralstelle für Denkmalschutz , Anm. d. Autorin] als berufene Hüterin der alten österreichischen Kulturtradition zu sehr vor der Geschichte verantwortlich, als daß sie nicht alles aufbieten würde, um die wenigen letzten Zeugen der Wiener Vergangenheit vor dem Untergange zu retten." 65

Nicht zuletzt versuchte man auch mit dem Verweis auf den wirtschaftlichen Faktor des Städtetourismus und der dafür notwendigen Erhaltung „Alt-Wiens“ ein Einlenken der Stadtverwaltung zu erwirken, denn die Anziehungskraft Wiens liege „in jenem geschichtlich gewordenen Stimmungswert“, der erst „in der Gesamtheit ihrer Bauten “ in Erscheinung trete. ${ }^{66} \mathrm{Im}$ Laufe der 1930er-Jahre wurde vonseiten der Zentralstelle eine prinzipielle Klärung und Besprechung der aktuellen Fragen der Stadtregulierung mehrfach angeregt, um endlich eine für beide Seiten befriedigende Lösung des seit Jahren schwelenden Konfliktes zu erzielen. ${ }^{67}$ Bereits Ende der 1920er-Jahre hatte man eine notwendige Absprache und Übereinkunft zwischen den Organen der Stadt und der Zentralstelle für Denkmalschutz gefordert. ${ }^{68}$ Die Forderungen wurden nun zunehmend dringlicher. 1936 sprach man in der Zeitschrift Deutsche Kunst und Denkmalpflege etwa die Bitte an Bürgermeister Richard Schmitz aus, die „Regulierungspläne einstampfen und neue unter Bedachtnahme auf die ehrwürdigen Zeugen unserer stolzesten und herrlichsten Kulturblüte ausarbeiten zu lassen“.69

In den folgenden Jahren scheint allerdings kein Einvernehmen gefunden worden zu sein. In einer Besprechung im September 1937, anlässlich der Demolierungen in der Inneren Stadt, kam es im Palais Paar „zu einer sehr heftigen Stellungnahme der Bauwerber und auch verschiedener Vertreter des Magistrates“, wobei der Vorsitzende forderte, dass endlich eine „prinzipielle Einigung zwischen d. Magistrat u. der Z.St.f. D. zu Stande kommen möge, um die immer wieder auftauchenden Schwierigkeiten zu beseitigen“. Es folgte der Vorschlag, den noch immer geltenden Regulierungsplan von 1892 „einer Prüfung zu unterziehen und für die Zukunft beiderseits bindende Richtlinien auszuarbeiten“.70

Kritik am Generalregulierungsplan hatten bis dahin nicht nur die Beamten der Zentralstelle geübt, auch die kritischen Stimmen aus der Architektenschaft mehrten sich zunehmend. Die Zentralvereinigung der Architekten Österreichs unter der Führung von Clemens Holzmeister forderte im Oktober 1937 in einem Schreiben an den Bürgermeister, dringend den Flächenwidmungs- und Bebauungsplan für Wien ,in seiner gesamten Ausdehnung neu zu bearbeiten“.71 Die oftmals radikalen Regulierungsabsichten des Stadtbauamtes, die man in Fortführung der Pläne aus der Monarchie und im Sinne des „schöpferischen Tätigwerdens“ umzusetzen versuchte, stießen mit fortwährender Dauer der Assanierungsaktion, auch in künstlerischen und stadtplanerischen Kreisen, zunehmend auf Kritik. Diese galt nicht nur der haltlosen Zerstörung ganzer Altstadtbereiche, sondern auch der Tatsache, dass man dafür weiterhin einer veralteten Verkehrsplanung folgte, die den Regulierungsplan zur Inneren Stadt in den 1890er-Jahren unter noch ganz anderen Voraussetzungen und verkehrstechnischen Gegebenheiten erstellt hatte. Auch Kunsthistoriker und Denkmalpfleger sprachen sich 
für eine Überarbeitung aus, da er in einer Zeit erstellt worden war, als man „das Barock noch hässlich fand“72, weshalb darin auf die oftmals weit in den Straßenraum vorragenden Gebäude dieser Bauperiode auch keinerlei Rücksicht genommen wurde.

Nach jahrelangen Debatten um die Überarbeitung des Generalregulierungsplanes und auch als Reaktion auf die wachsende Kritik aus der Öffentlichkeit und der Architektenschaft verfügte Bürgermeister Richard Schmitz letztlich im November 1937 die Bildung einer Kommission, die den Generalstadtplan „auf seine älteren Regulierungsideen hin durchsehen“ solle, um „zu weitgehende Umgestaltungsvorschläge auszumerzen“ und „das schützenswerte Alte“ in Form von Schutzzonen einzugrenzen. Die Bildung der Kommission hatte Schmitz auf Anraten von Stadtbaudirektor Musil angeordnet, da dies seiner Ansicht nach „für die Bevölkerung beruhigend wirken“ werde. ${ }^{\mathbf{7 3}}$ Zur Umsetzung kam es - vermutlich infolge der politischen Veränderungen im Frühjahr 1938 und des „Anschlusses“ an das Deutsche Reich - nicht mehr. Die Vorarbeiten reichten nur bis zur Nennung „geeigneter“ Fachleute, zu denen Musil unter anderem Karl Holey, Clemens Holzmeister und Emmerich Siegris zählte, und zum Entwurf einer Geschäftsordnung. ${ }^{\mathbf{7 4}}$

Im Laufe des Spätjahres 1937 kann also von einem Umdenken in der Stadtverwaltung gesprochen werden. ${ }^{\mathbf{7 5}}$ Auch die regierungsnahen Tageszeitungen präsentierten Bürgermeister Richard Schmitz nun als „Retter von Alt-Wien“ und lobten seinen Einsatz für das Wiener Stadtbild. ${ }^{\mathbf{7 6}}$ Die Darstellung seiner Person in der Öffentlichkeit wandelte sich somit vom „Arbeitsbeschaffer“ und Förderer der Bauwirtschaft zu Beginn der Assanierungsaktion zum obersten Befürworter der Stadterhaltung kurz vor dem „Anschluss“. Der Sinneswandel und die Entscheidung, eine Kommission zur Überarbeitung des Generalregulierungsplanes einzuberufen, wurden von der Zentralstelle jedenfalls begrüßt. ${ }^{77}$ Auch Karl Giannoni, Leiter der Fachstelle für Fragen des Naturund Heimatschutzes in der Zentralstelle für Denkmalschutz, zeigte sich erfreut. ${ }^{\mathbf{7 8}}$

$\mathrm{Zu}$ einer Aussprache zwischen der Zentralstelle und der Gemeinde Wien in Fragen des Denkmal- und Stadtbildschutzes scheint es schließlich im Oktober 1938 gekommen zu sein, ${ }^{\mathbf{7 9}}$ nachdem die Kämpfe um eine „Gesamtregelung“ über 30 Jahre lang die Wiener Stadtplanung geprägt hatten. ${ }^{\mathbf{8 0}}$ Die Zentralstelle beabsichtigte, sich mit dem Stadtbauamt zu einigen, welche Bauten und Stadtteile aufgrund „neuzeitlicher Erfordernisse" umzubauen oder zu demolieren wären, und solche Baulichkeiten und Stadtteile festzulegen, die „unter allen Umständen“ erhalten bleiben sollten. ${ }^{\mathbf{8 1}}$ Das Treffen und die weitere Zusammenarbeit verliefen offenbar harmonisch, denn im Jänner 1939 verkündete die Zentralstelle schließlich selbstbewusst, dass man nunmehr, „in weit höherem Maße als in der vergangenen Zeit Einfluss auf die Gestaltung des Wiener Stadtbildes“ nehmen könne und für die Zukunft „keinerlei Gefahren für das Wiener Stadtbild und seine historischen Bauten zu erwarten" seien.82

\subsection{Beobachtungen zur denkmalfachlichen Bewertung}

Der Wert der stadtbildprägenden Altbauten, die jedoch nicht den Rang eines Einzeldenkmals erreichten, wird in den Akten häufig betont. Warum aber konnte man nicht zumindest in einigen wenigen Fällen für eine Erhaltung argumentieren und einen Denkmalstatus erwirken? Welche Kriterien gab es für eine Unterschutzstellung, welche Denkmalwerte waren ausschlaggebend? Aufschluss geben hier - mehr als das Denkmalschutzgesetz in der damaligen Fassung - die Bewertungen der zum Abriss 
vorgesehenen Gebäude durch die Referenten der Zentralstelle. Nachdem das Kuratorium des Assanierungsfonds einen Antrag auf Subvention eines Abrissvorhabens und Neubaus bewilligt hatte, wurde die Zentralstelle für Denkmalschutz in einem offenbar rein formalen Schritt um prinzipielle Stellungnahme gebeten. Durch den Referenten erfolgte nach dem Lokalaugenschein eine Bewertung des Objektes auf Basis des Denkmalschutzgesetzes und der damals geltenden Denkmaleigenschaften, die ein schützenswertes Gebäude zu erfüllen hatte.

Das Denkmalschutzgesetz führte - mit einem ähnlichen Wortlaut wie heute - geschichtliche, künstlerische und kulturelle Gründe an, weshalb die Erhaltung eines Denkmals im öffentlichen Interesse liegen konnte. Zahlreichen zum Abriss bestimmten Gebäuden bescheinigte man eine geringe kunst- und architekturhistorische Bedeutung. Die Bewertung umfasste oftmals die Schlagworte „künstlerisch belanglos“ oder sogar „wertlos“.83 Diese Einschätzungen sind heute bei vielen Objekten kaum nachvollziehbar, einerseits, weil es sich meist zwar um künstlerisch nur gering gestaltete Objekte, aber dennoch um Gebäude aus dem 18. und frühen 19. Jahrhundert handelte, die zum Ermessenszeitpunkt bereits rund 100 bis 150 Jahre oder teilweise noch älter waren und auch einen nicht unwesentlichen historischen Wert besaßen. Andererseits waren die theoretischen Fundamente der „modernen Denkmalpflege“, die jedes Objekt als künstlerischen Ausdruck der jeweiligen Zeit verstand, zu Beginn des 20. Jahrhunderts in Wien gelegt worden und hätten zumindest eine differenzierte Analyse erwarten lassen.

Alois Riegl hatte 1903 in seiner wegweisenden Schrift Der moderne Denkmalkultus. Sein Wesen, seine Entstehung ein vielschichtiges, verzweigtes Gefüge an Denkmalwerten erarbeitet - das bis heute nicht an Aktualität und Relevanz verlorene „Rüstzeug“ der Denkmalpflege. Einzelnen Werten, wie vor allem dem Alterswert, maß er dabei eine übergeordnete Bedeutung bei und schuf damit eine gewisse Hierarchie in der Berücksichtigung der Denkmalwerte. ${ }^{\mathbf{8 4}}$ Dem Alterswert, der laut Riegl Ausdruck in einer gewissen Unvollkommenheit und in den Spuren der Vergänglichkeit findet und der das Denkmal erst als Menschenwerk fassbar und in seiner Geschichtlichkeit erfahrbar macht, sagte er generell für die Denkmalpflege des 20. Jahrhunderts eine herausragende Bedeutung voraus. ${ }^{\mathbf{8 5}}$ Noch 1911 spricht Karl Giannoni vom Vorrücken des „entwicklungsgeschichtlichen Gedankens" in die Denkmalpflege und der zunehmenden Bedeutung des „durch seinen Stimmungsinhalt wirkenden gefühlsmäßigen Alterswerts" gegenüber dem wissenschaftlichen historischen Wert. ${ }^{\mathbf{8 6}}$ In der denkmalpflegerischen Praxis der 1930er-Jahre galt der Alterswert der Gebäude allerdings nicht als ausreichendes Kriterium, um ein Objekt unter Schutz zu stellen oder zumindest für seine Erhaltung zu plädieren. Dies geht aus den Bewertungen der Altbauten durch die Referenten der Zentralstelle deutlich hervor, die sehr oft den „malerischen“, „reiz-“ oder „stimmungsvollen“ Charakter der Bestandsbauten betonten, die Gebäude dann allerdings zum Abbruch freigaben. Als einzige Maßnahme forderte die Zentralstelle in diesen Fällen Fotografien der Altbauten ein, um zumindest die Erinnerung an die Bauten zu „konservieren“. Die dem Alterswert entsprechende malerische Stimmung in den Altstadtbereichen wurde zwar als Qualität wahrgenommen, aber nicht zum eigentlichen Denkmalwert erhoben.

Weitaus größere Bedeutung maß man hingegen der künstlerischen Ausführung und der kunsthistorischen Bedeutung der Gebäude bei. Ab November 1937 bemühte 


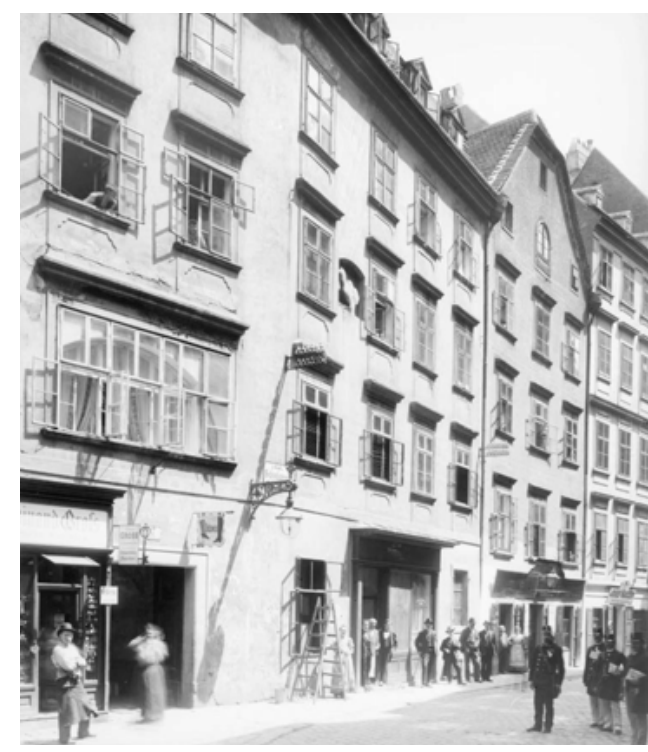

Abb. 61 August Stauda, Ansicht der Häuserzeile Schönlaterngasse 7-11, Aufnahme aus dem Jahr 1902

sich Karl Ginhart etwa um die Unterschutzstellung mehrerer Wohnhäuser in der Schönlaterngasse, ${ }^{\mathbf{8 7}}$ die „zu den baukünstlerisch und geschichtlich interessantesten Bürgerbauten Wiens" zählen und die „auch für das Gesamtbild des schönsten alten Stadtteiles der Stadt Wien von höchster Bedeutung“ seien (Abb. 61).88 Den angrenzenden Gebäuden bescheinigte man keinen künstlerischen Wert, weshalb eine Stellung unter Denkmalschutz nur „zum Zwecke der Erhaltung des altertümlichen Straßenbildes" infrage komme. ${ }^{89}$ Die Möglichkeit, charakteristische, zusammenhängende Ensembles zu schützen, wurde allerdings erst 1978 im österreichischen Denkmalschutzgesetz verankert.90

Zahlreiche Objekte aus der josephinischen Zeit und dem Biedermeier mit einer charakteristisch schlichten Fassadengestaltung mussten weichen, obwohl ihre Erhaltung zur Abbildung der historischen Entwicklung der Stadt und auch aus ästhetisch-atmosphärischen Gründen wesentlich gewesen wäre.91 Dies deckt sich auch mit der Feststellung von Frodl-Kraft, dass in der Riegl-Nachfolge unter Dvořák die Relativität seiner Wertskala und sein entwicklungsgeschichtlicher Ansatz nicht verstanden wurden.92 Dies setzte sich in den 1930er-Jahren offensichtlich fort. Die von Alois Riegl zu Beginn des Jahrhunderts betonte Relativität des Kunstwertes, also die Gleichwertigkeit des künstlerischen Ausdruckes und der künstlerischen Produktion aller Epochen, wurde in der denkmalpflegerischen Praxis nun „ernstlich in Frage gestellt“, mit Auswirkungen auf das „Ausleseprinzip“ der Denkmalpflege, wie Dagobert Frey im Jahr 1928 feststellte.93 Nicht alle künstlerischen Strömungen wurden gleichwertig behandelt und bewertet. Die bildende Kunst und Architektur des Barock - Letztere hatte der Generalregulierungsplan von 1892 noch völlig ignoriert - fanden zwar zunehmend Anerkennung,94 aber auch noch Mitte der 1930er-Jahre nicht immer die gebührende Wertschätzung.95 Der fortschreitende Verlust an Bauten dieser Zeit ging einher mit einer verstärkten kunsthistorischen Forschung, die in den 1930er-Jahren einen Höhepunkt erreichte. ${ }^{\mathbf{6}}$ Vor allem aber die Architektur des Historismus wurde in den 1930er-Jahren grundsätzlich gering geschätzt. ${ }^{\mathbf{9 7}}$ Kritisiert wurden in erster Linie historistisch „bekleidete“, mit Dekor „überladene“ Wohnhäuser. Bei Monumentalbauten wie der Staatsoper oder der Akademie der bildenden Künste lag die Sache freilich etwas anders. ${ }^{\mathbf{9 8}}$

Dem historischen Wert, den Riegl einem Objekt - unabhängig von seiner künstlerischen Ausführung - als Glied in der Entwicklung der Kunst- und Kulturgeschichte zusprach, wurde geringere Bedeutung beigemessen als dem Kunstwert. Die damals noch zahlreich vorhandenen Wohnhäuser aus der Zeit von 1750 bis 1850 kamen allerdings nur bei besonders ausgeprägtem Kunstwert für Unterschutzstellungen infrage. ${ }^{99}$ Das Objekt Althangasse 23, ein Gebäude aus dem 18. Jahrhundert und das „letzte Haus aus alter Zeit“ in der ehemaligen Vorstadt Liechtenthal, wurde 1928 demoliert. Obwohl 
es zuvor als „historisches Wahrzeichen“ bezeichnet und als Überrest einer ehemaligen Vorstadtbebauung auch mit einem historischen Wert belegt worden war, wurde vonseiten der Zentralstelle, aufgrund des ausschließlich historischen Wertes, die Abrissbewilligung für das angeblich baufällige Gebäude erteilt.100 Ebenfalls bereits in den 1920er-Jahren wurde auch das „letzte Doppelgiebelhaus der Marktgasse“ (Haus Nr. 26) - nach ähnlicher Argumentation - abgetragen. ${ }^{101}$

In der denkmalpflegerischen Praxis der 1930er-Jahre war demnach der Kunstwert bestimmend. Weniger Bedeutung hatte das Alter des Gebäudes, seine Bedeutung für das Stadtbild oder seine historische Relevanz, etwa für die Stadtbau- und Architekturgeschichte. Bei unzureichender künstlerischer Qualität wurde dem Abriss zugestimmt. Selbst wenn einem Gebäude durch den Referenten ein „Stimmungswert“ (Alterswert) bescheinigt wurde, war es dennoch von der kunsthistorischen Bedeutung abhängig, ob eine Erhaltung gerechtfertigt erschien. ${ }^{\mathbf{1 0 2}}$ Untermauert wird dies auch durch den Vergleich des Denkmalschutzgesetzes von 1923 mit der Neufassung des Statuts vom 31. Dezember 1936 und dem darin leicht geänderten Wortlaut zu den Denkmaleigenschaften, denn laut Statut standen Denkmale aufgrund ihrer „geschichtlichen, kulturgeschichtlichen oder kunstgeschichtlichen Bedeutung oder wegen ihres ästhetischen Wertes im öffentlichen Interesse“.103 Eine besondere Relevanz wird damit in der Bewertung von Baudenkmalen einmal mehr den ästhetischen, den architektonischen und künstlerischen Qualitäten eines Objektes zugesprochen.

Es passt daher auch durchaus ins Bild, dass die Zentralstelle bei vielen Objekten die Rettung wertvoller „Versatzstücke“, wie Fensterrahmen, Portale, Skulpturenschmuck oder Stuckdecken, und häufig auch ihre Wiederverwendung im Neubau veranlass te. ${ }^{\mathbf{1 0 4}}$ Ausstattung und Dekorelemente wurden demnach nicht als mit dem Gebäude verbundener Teil eines Ganzen betrachtet, sondern als einzige davon erhaltenswerte Bauteile. ${ }^{105}$ Die „spolienartige“ Weiterverwendung in den Neubauten wurde in einigen Fällen als Auflage für die Abtragungsbewilligung genannt. Diese Versatzstücke sollten offenbar die Geschichtlichkeit des Ortes verdeutlichen und zumindest die Erinnerung an den Vorgängerbau bewahren. ${ }^{106}$ So veranlasste man etwa die Rettung einiger Kunstgegenstände aus dem Palais Paar in der Wollzeile und ließ dort unter restauratorischer Aufsicht mehrere Stuckmedaillons des Marstalles abnehmen, um sie vor der Zerstörung zu bewahren. ${ }^{107}$

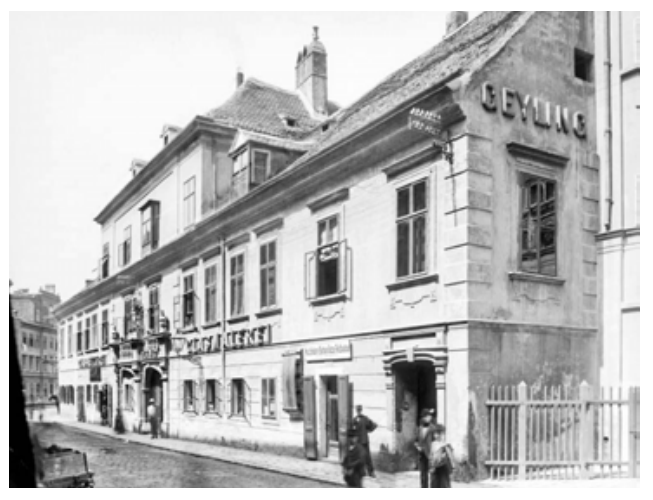

Abb. 62 August Stauda, Ansicht des „Geylinghauses“, Windmühlgasse 28, 1899
Neben den Denkmalwerten nach Alois Riegl waren auch noch andere Kriterien für eine Schutzwürdigkeit entscheidend. Auch der Erhaltungszustand wurde mitberücksichtigt, obwohl das Denkmalschutzgesetz von 1923 diesbezüglich keine Einschränkung äußerte. Der schlechte Zustand war mitunter ausschlaggebend, dass ein Gebäude als nicht schutzwürdig befunden wurde. ${ }^{\mathbf{1 0 8}}$ Daneben wurden auch die städtebaulichen Verhältnisse bei Unterschutzstellungsfragen in die Überlegungen miteinbezogen. Durch die in der Spätgründerzeit zurückversetzte Baulinie und 


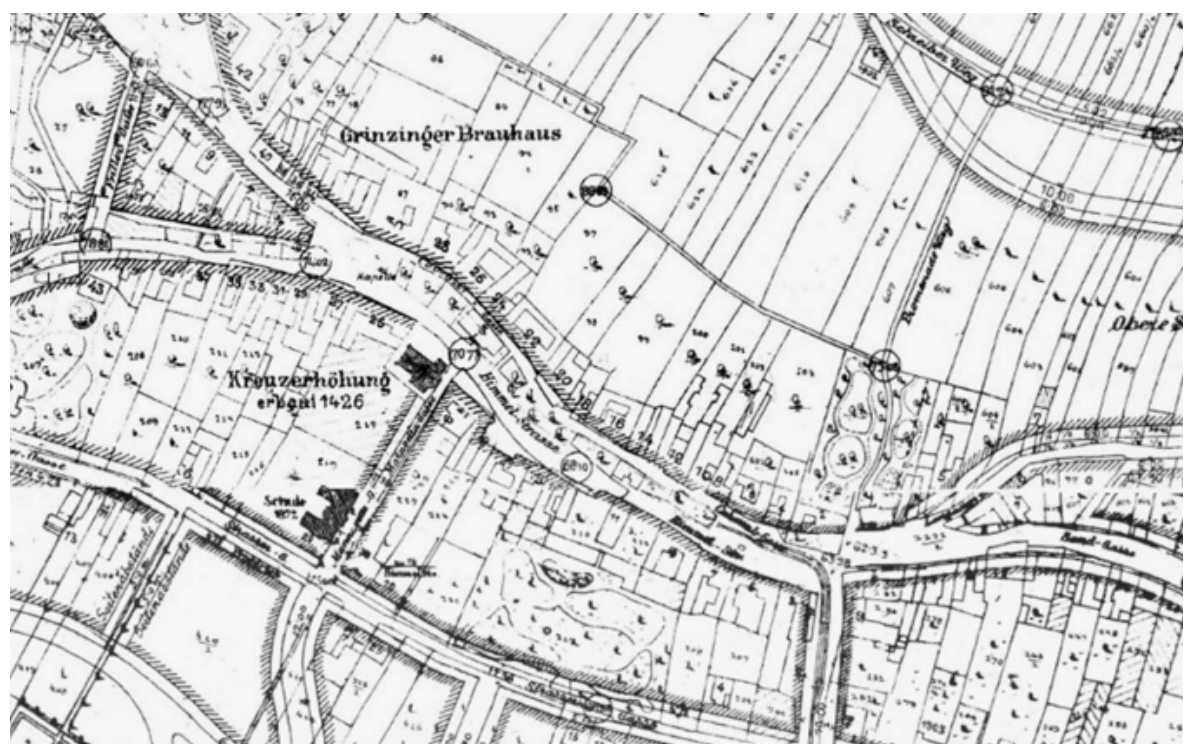

Abb. 63 Generalstadtplan Wien, Ausschnitt Grinzing, Regulierung im Bereich der Himmelstraße und Sandgasse, Fassung 1912

den Austausch der Bebauung ragte der verbliebene Altbestand häufig „störend“ in den Straßenraum hinein. Dass damit auch eine Einzigartigkeit und (lokal-)historische Bedeutung der Objekte gegeben war, wurde nicht als Grund für eine Unterschutzstellung angesehen. Dem architektonisch durchaus bemerkenswerten „Geylinghaus“ (Windmühlgasse 28, 6. Bezirk; Abb. 62) wurde im Jahr 1936 der Denkmalschutzstatus explizit deshalb nicht gewährt, da ein Abriss aus verkehrstechnischen Gründen ohnehin zu erwarten war. ${ }^{\mathbf{1 0 9}}$ Das (öffentliche) Interesse an der Erhaltung eines Gebäudes musste also häufig auch hinter die Bedürfnisse des Verkehrs zurücktreten:

„Bei aller Würdigung der historischen und künstlerischen Bedeutung [...] kann die Z.St.f. D.Sch. sich nicht der Tatsache verschließen, daß das Gebäude sehr weit über die neue Baulinie vorragt und daher leider zu einem großen Verkehrshindernis [...] wurde. In Anbetracht dieses Umstandes wurde daher h. a. von der erbetenen Stellung des Hauses unter die Best. des Bundesgesetzes vom 25. Sept. 1923 [...] abgesehen, weil die Z.St. f. D.Sch. mit Rücksicht auf die Forderungen des modernen Großstadt-Verkehrs in naher Zukunft vor die zwingende Notwendigkeit gestellt werden würde, den Standpunkt das Haus unbedingt zu erhalten wieder aufzugeben.“110

\subsection{Ein Stück „Alt-Wien“ gerettet}

In einigen wenigen Fällen trat die Zentralstelle sehr vehement für eine Erhaltung ein, wie im Falle des Ortsbildes von Grinzing und der Griechengasse und konnte schließlich auch ihre Interessen durchsetzen.

Die Regulierung des Ortskerns von Grinzing wurde Mitte der 1930er-Jahre zu einer dringlichen Frage, denn der zunehmende Verkehr auf den Kahlenberg - über die neu geschaffene Höhenstraße - sollte durch Grinzing hindurch oder daran vorbei geschleust werden. Im Frühjahr 1937 wurde ein Architekturwettbewerb zur verkehrstechnischen 
Regulierung unter Erhaltung des Grinzinger Ortsbildes ausgeschrieben. Das Stadtbauamt versicherte, dass man „die weitestgehende Schonung dieses wichtigen Stadtbildes im vollen Einvernehmen mit der Oeffentlichkeit"111 anstrebe. Ende des Jahres 1937 kündigte Bürgermeister Schmitz die Erhaltung der baulichen Struktur der Wiener Vororte sowie die Überarbeitung des veralteten Regulierungsplanes an (Abb. 63).112 Den noch gültigen Plan für Grinzing aus der Zeit der Eingemeindung, der sich bei Umsetzung auf das Ortsbild massiv ausgewirkt hätte, hatte man bereits über-

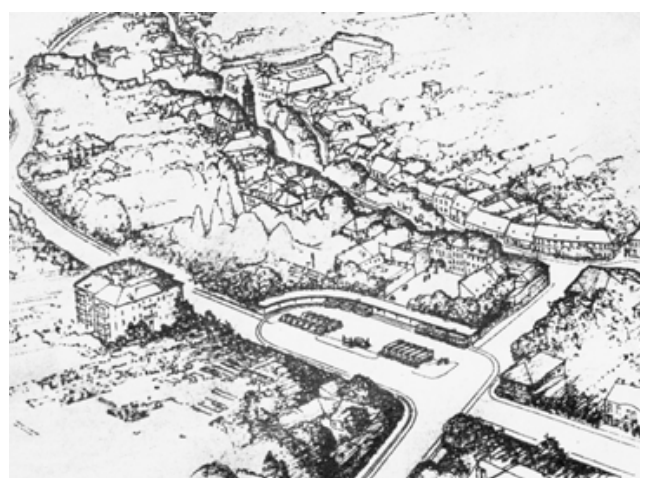

Abb. 64 Egon Fridinger, Umfahrungsstraße in Grinzing, Tageszeitung Das interessante Blatt, Jänner 1938 arbeitet. Der Wettbewerb sollte den neuen Baulinienplan nun überprüfen und eine das Ortsbild „schonende“ Lösung bringen. ${ }^{\mathbf{1 1 3}}$ Die Zentralstelle war sehr um „die Erhaltung des alten reizvollen Weinbauortes Grinzing “ bemüht und versuchte daher, den Bau einer Umfahrungsstraße zu erwirken. ${ }^{\mathbf{1 1 4}}$ Da keiner der Entwürfe dem Preisgericht zur Gänze zusagte, brachte der Wettbewerb Ende des Jahres 1937 für die Entwürfe der Architekten Egon Friedinger und Rudolf Perthen (Abb. 64) zwei zweite Plätze.115 Beide Preisträger sahen die von der Zentralstelle geforderte Umfahrungsstraße vor. ${ }^{\mathbf{1 1 6}}$

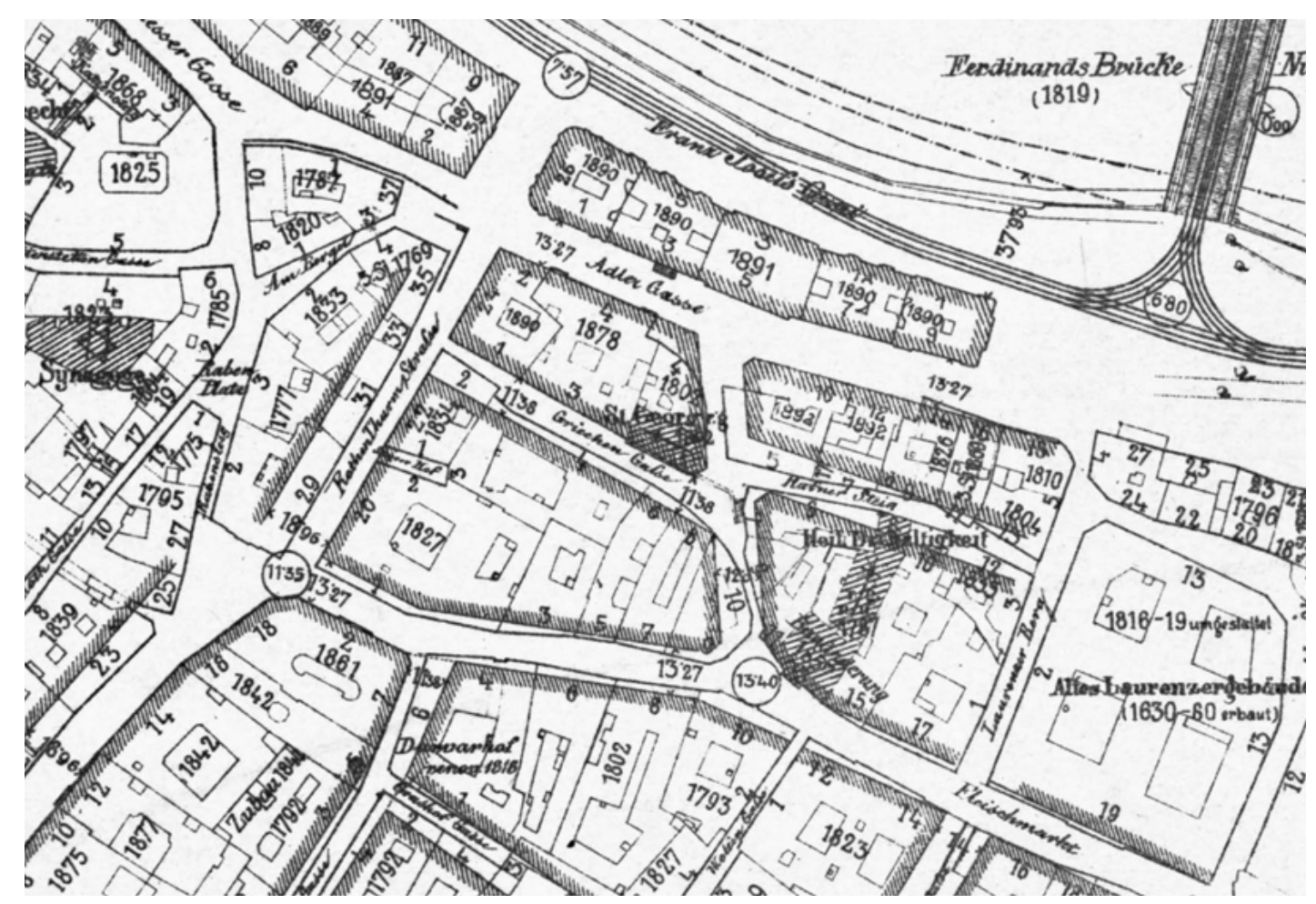

Abb. 65 Generalregulierungsplan, Ausschnitt des Altstadtbereiches zwischen Laurenzerberg und Rotenturmstraße; zeigt u.a. die geplante Regulierung der Griechengasse, 1892 


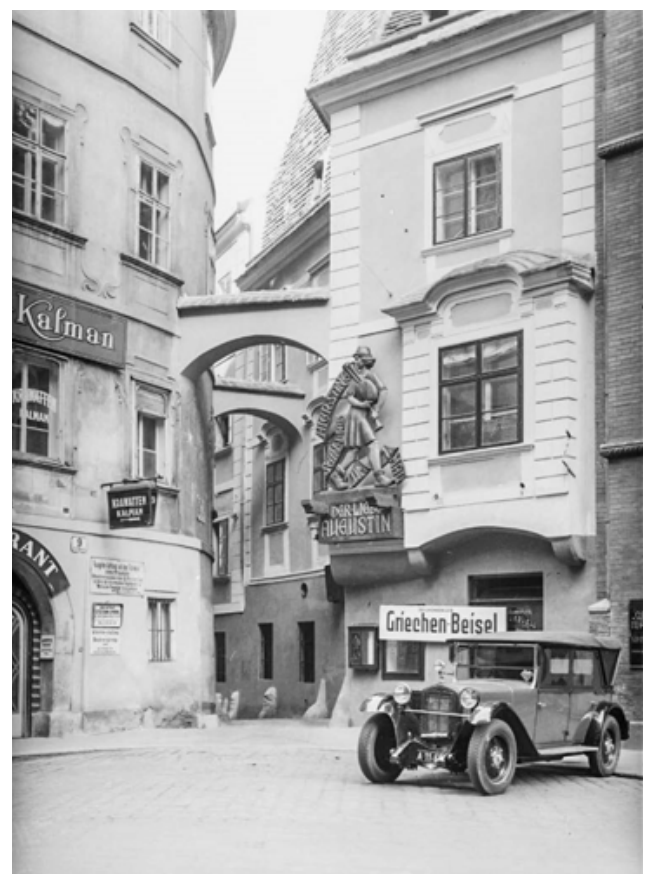

Abb. 66 Einblick in die Griechengasse mit den ursprünglich zu regulierenden Hausnummern 10 und 11, 1935

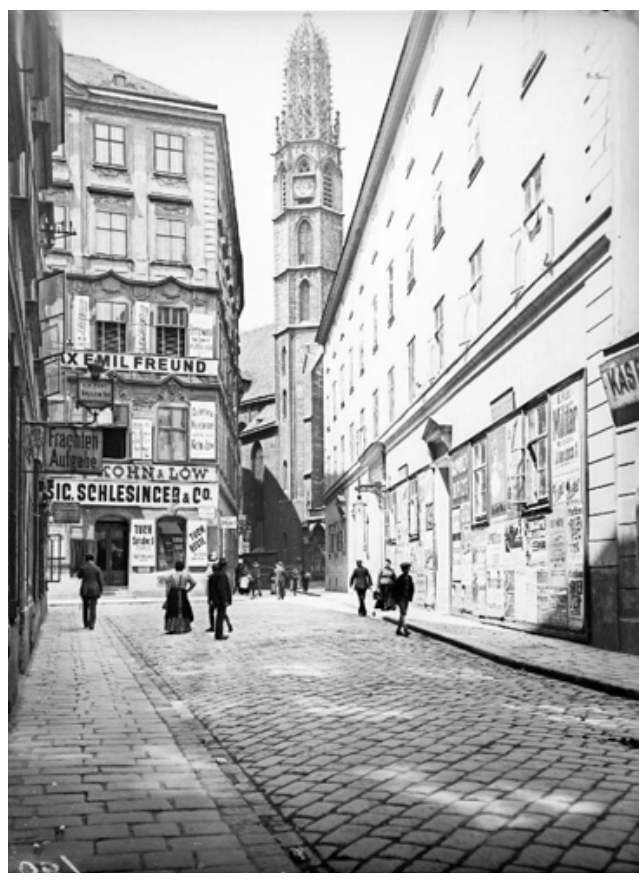

Abb. 67 Salvatorgasse, Blick von Südosten gegen Stoß im Himmel und Maria am Gestade, 1911

Hätte man den Generalregulierungsplan zur Inneren Stadt umgesetzt, wären durch die rigide Baulinienführung des Stadtbauamtes auch mehrere, zum Teil denkmalgeschützte Gebäude entlang der Griechengasse in der Inneren Stadt zerstört worden (Abb. 65). Schon in der Österreichischen Kunsttopographie wurde die Bedeutung der Griechengasse, die „eines der altertümlichsten Stadtbilder Wiens“117 aufweise, unterstrichen. Gerade die durch einen Schwibbogen verbundenen beiden Gebäude am Eingang der Gasse würden „eine der altertümlichsten und malerischsten Baugruppen der Stadt“118 bilden (Abb.66). Am 5. Februar 1938 teilte die Stadtverwaltung der Zentralstelle die auf Verfügung von Bürgermeister Schmitz beschlossene Änderung der Baulinien mit, ${ }^{119}$ die im Bereich des malerischen Engpasses der Griechengasse den Bestand nun völlig unberührt ließen. ${ }^{\mathbf{1 2 0}}$ Diesen Erfolg führte Referent Siegris auf seine wiederholt energisch vorgebrachte Kritik am bestehenden Regulierungsplan zurück. ${ }^{\mathbf{1 2 1}}$ Letztlich war es aber wohl die zunehmende Kritik am rasanten Stadtumbau von Fachleuten und aus der Bevölkerung, die hier ein Einlenken bewirkte.

Letztendlich konnte die Zentralstelle nur in wenigen Fällen, vor allem in der Inneren Stadt, wo auch die mediale Aufmerksamkeit stärker gegeben war, eine Erhaltung erwirken und sich gegen die Regulierungsabsichten der Stadtverwaltung durchsetzen. Einige Einsprüche der Zentralstelle gegen Demolierungs- und Bauvorhaben verwiesen dabei auf die Bedeutung der bestehenden Bausubstanz für den Ensemblecharakter und das Stadtbild. So konnte etwa die Erhaltung des Palais Eskeles in der Innenstadt erfolgreich durchgesetzt werden. Das Palais mit klassizistischer Fassade wurde zwar vom Referenten als „wertlos“ eingestuft, doch die drohende Lücke im Straßenbild wäre absolut störend und „unschön“ gewesen, weshalb man sich gegen den Abriss 
einsetzte.122 Erfolgreich bekämpft wurde beispielsweise auch die Demolierung des Gebäudes Stoß im Himmel 3 am Passauer Platz, nahe der Kirche Maria am Gestade (Abb. 67). Das als „eines der bemerkenswertesten Häuser aus dem 18. Jahrhundert“ bezeichnete Objekt befand Referent Siegris 1935 als „wichtigen Teil einer ganzen Wand des Passauerplatzes und der Kirche“, dessen Demolierung „einen schweren Verlust für den Kirchenplatz und für das reizvolle Wr. [Wiener , Anm. d. Autorin] Stadtbild“ bedeutet hätte. ${ }^{123}$ Die Charakteristik und Bedeutung eines Gebäudes für das Stadtbild wurden also im Einzelfall als wichtige Eigenschaften, aber nur bei künstlerisch bedeutsamen Objekten als ergänzender Denkmalwert angeführt. 
1 „Der Umbau des Palais Herberstein durch Arch. Ing. Felix Nemečić“", in: Österreichische Kunst, 8. Jg., H. 1, 1937, S. 19. 2 BDA-Archiv, Top. Mat., Wien I. Bezirk, Profan, Schauflerg. 2, 590/Dsch/1937, Av Emmerich Siegris, 16. 2.1937. 3 „Umbau am Michaelerplatz“, in: Profil, 4. Jg. H. 12, 1936, S. 570. 4 Bei diesen Objekten handelte es sich um die Adressen Tiefer Graben 36 und Am Gestade 8. 5 Magistrat der Stadt Wien (Hrsg.), Kunstförderung durch die Stadt Wien. Aufträge und Erwerbungen von Werken der Kunst und des Kunstgewerbes unter dem Bürgermeis ter Richard Schmitz in den Jahren 1934-1936 (Wien im Aufbau), Wien 1937c, S.6. 6 „Maria am Gestade. Die städtebauliche Lösung des Geländes vor der Kirche“, in: Die Pause, 3. Jg., H. 1/2, 1938, S. 48-52. 7 K. K. ZentralKommission für Kunst- und Historische Denkmale (Hrsg.) Österreichische Kunsttopographie, Bd. 15, Kunsthistorischer Atlas der k. k. Reichshaupt- und Residenzstadt Wien und Verzeichnis der erhaltenswerten historischen, Kunst- und Naturdenkmale des Wiener Stadtbildes, Wien 1916 S. 49. 8 Karl Ginhart, „Kurzberichte. Wien“, in: Deutsche Kunst und Denkmalpflege, 3. Jg., 1936, S.303-305, hier S. 304. 9 Ebd., S. 303. 10 Zu erwähnen ist in diesem Zusammenhang vor allem: Cornelius Gurlitt, Handbuch des Städtebaues, Berlin 1920, S. 247-248; Karl Kühn, Die schöne Altstadt. Ihr Schutz, ihr Umbau, ihre Verkehrs verbesserung. Eine Untersuchung zur praktischen Auswertung der Erkenntnis vom Wesen der alten Stadt, Berlin 1932, S. 37-44. Werner Lindner, Vorsitzender des Deutschen Bundes Heimatschutz, schlägt Arkaden und Laubengänge vor, um Abbrüche in Altstädten zu vermeiden: ders., Die Stadt. Ihre Pflege und Gestaltung (Die landschaftlichen Grundlagen des deutschen Bauschaffens, 2), München 1939, S.172. 11 Siehe dazu: „Carl Wilhelm Schmidt. Bauten“, in: Österreichische Kunst, 7. Jg., H. 12 , 1936, S. 5-17. 12 Erste Überlegungen dazu wurden sogar schon um 1900 angestellt. Siehe dazu die Akten im BDA-Archiv, Top. Mat., Wien I. Bezirk, Profan, Wipplingerstraße 7, 5432/Dsch/1932 und 627/Dsch/1933. 13 LGBI. für Wien 11/1930 (Bauordnung für Wien), § 87 Abs. 1. Die Inhalte des Paragrafen sind in der heutigen Bauordnung in abgeänderter bzw. ergänzter Form in $\S 85$ enthalten. 14 LGBI. für Wien 11/1930 (Bauordnung für Wien), § 87 Abs. 3. 15 Die Möglichkeit, charakteristische, zusammenhängende Ensembles zu schützen, wurde erst 1978 im österreichischen Denkmalschutzgesetz verankert: BGBI. 167/1978; siehe dazu u.a.: Bundesdenkmalamt (Hrsg.), Denkmalpflege in Österreich, Wien 1990, S.146. Auch die Einrichtung von Schutzzonen und die rechtliche Verankerung des Ortsbildschutzes sollte in Wien noch fast 40 Jahre in Anspruch nehmen (Altstadterhaltungs novelle der Bauordnung für Wien im Jahr 1972, LGBI. für Wien 16/1972). 16 BGBI Nr. 533/1923, § 8. 17 Leodegar Petrin, „Die Organisation der Denkmalpflege in Österreich“, in: Deutsche Kunst und Denkmalpflege, 4. Jg., 1937, S. 59-61, hier S. 60. 18 Wilfried Kirsch, „Vom Werden des Österreichischen Heimat- und Naturschutzes", in: Deutsche Kunst und Denkmalpflege, 5.Jg., 1938, S. 62-64, hier S. 63. 19 Dies wird aus den Standardschreiben des Stadtbauamtes/Assanierungsfonds deutlich: „Im Sinne des Übereinkommens wird um umgehende prinzipielle Stellungnahme vom Standpunkte des Denk malschutzes ersucht." Siehe z. B.: BDA-Archiv, Top. Mat. Wien I. Bezirk, Profan, Singerstraße 4, 2397/Dsch/1935. 20 BGBI. 67/1936, Bundesverfassungsgesetz, betreffend die Organisation des Denkmalschutzes im Bereiche der Länder und der Stadt Wien, §1. 21 Eva Frodl-Kraft, Gefährdetes Erbe. Österreichs Denkmalschutz und Denkmalpflege 1918-1945 im Prisma der Zeitgeschichte (Studien zu Denkmalschutz und Denkmalpflege, 16), Wien u.a. 1997, S. 111. 22 So festgelegt im LGBI. für Wien $43 / 1934$ betreffend die Errichtung eines Fonds zur Förderung der Bautätigkeit in Wien (Wiener Assanierungsfonds), § 2 . 23 „Fort mit den Verkehrshindernissen in Wien. Ein Jahr Arbeit für 40.000 Arbeitslose“, in: Neues Wiener Journal, 20.9.1934, S.3. 24 „Die Ausführung dieser Ideen wird sicher viele baukünstlerische Aufgaben stellen, deren Lösung zur Verschönerung unserer Vaterstadt und damit auch zur Belebung des Fremdenverkehrs beitragen wird." Siehe dazu: Wiener Stadtbauamt (Hrsg.), Hundert Jahre Wiener Stadtbauamt 1835-1935, Wien 1935, S.157. 25 Ebd. 26 BDA-Archiv, Top. Mat., Wollzeile 30, 3628/ Dsch/1937, Antwortschreiben des Stadtbauamtes auf einen Brief der Zentralstelle, 4.10.1937, S. 3. 27 „Arbeitsbeschaffung und Baugewerbe“, in: Der Baumeister, 1. Jg., Folge 31, 1.11.1934, S.1-2, hier S. 2. 28 Ebd. 29 Franz Musil war Ingenieur und Verkehrsplaner und hatte Erfahrung im Bau von Untergrundbahnen. 1917 trat er in das Wiener Stadtbauamt ein, war dort bis 1921 für Schnellbahnen zuständig und daran anschließend in der Abteilung für technisches Verkehrswesen beschäftigt. Im Jänner 1925 übernahm er die Leitung des Stadtbauamtes. In seine Amtsperiode fiel somit auch das Wohnbauprogramm des Roten Wien. Im Jahr 1934 wurde er nicht nur in seinem Amt bestätigt, das er bis 1941 innehatte, sondern sein Einfluss auch wesentlich erweitert. Zur Biografie siehe: Wiener Stadtbauamt 1935, S. 54-55. 30 WStLA, 1.5.4. Stadtbaudirektion, A1, Allg. Reg., Schachtel 110/1938, GZ 236/1938, Brief Stadtbaudirektor Franz Musil an Bgm. Richard Schmitz, 26.1.1938. 31 Ebd., Schachtel 110/1938, GZ 236/1938, Brief Stadtbaudirektor Franz Musil an Bgm. Richard Schmitz, 26.1.1938. 32 Ebd., Schachtel 109/1937, GZ 4587/1937, Schreiben Stadtbaudirektor Franz Musil an den Magistratsdirektor, 29.11.1937. 33 Ebd., Schachtel 109/1937, GZ 4353/1937, Schreiben Stadtbaudirektor Franz Musil an Bgm. Richard Schmitz, 15.12.1937. 34 Ebd., Schachtel 109/1937, GZ 4353/1937, Antwortschreiben Stadtbaudirektor Franz Musil an die ZV, 29.12.1937. 35 Ebd., Schachtel 102/1936, GZ 3202/1936, Schreiben Stadtbaudirektor Franz Musil an Bgm. Richard Schmitz, 10.8.1936. 36 So die Selbstbezeichnung, siehe: BDAArchiv, Top. Mat., Wien I. Bezirk, Profan, Wollzeile 32, 3628/Dsch/1937, Antwortschreiben der Zentralstelle an den Oberstadtbaurat, 28.10.1937. 37 Frodl-Kraft 1997, S. 109. Festgehalten wurde die Neuorganisation im Bundesgesetz von 1934: BGBI. 47/1934 betreffend die Auflassung des Bundesdenkmalamtes; und im bereits erwähnten Bundesverfassungsgesetz 67/1936, das die Organisation der Denkmalpflege in den Bundesländern regelte. 38 Petrin galt als „willfähriger Beamter“. Siehe: Frodl-Kraft 1997, S. 112. Er war Jurist und ab 1905 im Bundesministerium für Kultur und Unterricht tätig. Bereits 1931 war er zum Präsidenten des damals noch bestehen- 
den BDA ernannt worden. Siehe: Theodor Brückler, Ulrike Nimeth, Personenlexikon zur österreichischen Denkmalpflege, Wien 2001, S. 201. Ab 1935 war er überdies Sektionschef des Bereichs „Kunst und Volksbildung“, bis man inn im September 1938 zwangspensionierte. 39 Petrin 1937, S. 59-61. 40 Ebd., S. 60. Clemens Holzmeister, Karl Holey und Karl Kraus bildeten 1937 den „Beirat für Fragen des Bauwesens auf dem Gebiet der Denkmalpflege“. $41 \mathrm{Vgl}$. etwa: Ernst Bacher, „150 Jahre Denkmalpflege in Österreich“, in: ÖZKD, Sonderheft, 54. Jg., H. 4, 2000, S. 460-465, hier S. 462.42 Frodl-Kraft 1997, S. 107. 43 Otto Demus, „Die österreichische Denkmalpflege“, in: Egon Loebenstein (Hrsg.), 100 Jahre Unterrichtsministerium 1848-1948, Festschrift des Bundesministeriums für Unterricht in Wien, Wien 1948, S. 393-411, hier S. 404. 44 Bacher 2000, S. 462.45 Frodl-Kraft 1997, S. 80-82. Auch Ernst Bacher verweist in diesem Zusammenhang auf die schlechte finanzielle und personelle Situation: Bacher 2000, S. 462 . 46 Frodl-Kraft 1997, S.64. 47 Bundesdenkmalamt 1990, S. 145-146. In diesem Zusammenhang sei auch auf den 2.Band des Dehio-Handbuches der Kunstdenkmäler Österreichs verwiesen, der 1935 erschien: Dagobert Frey (Hrsg.), Handbuch der deutschen Kunstdenkmäler. Österreich [hrsg. von Georg Dehio], Bd. 2, Wien, Niederösterreich, Oberösterreich und Burgenland, Berlin/Wien 1935. 48 Frodl-Kraft 1997, S.68. 49 BDAArchiv, Personalakt Karl Ginhart, 3606/Dsch/1936, Av, vermutlich November 1936. 50 BDA-Archiv, Personalakt Otto Demus, 3523/Dsch/1936 und 3657/Dsch/1936. 51 BDA-Archiv, Heimatschutz, Karton 3a, Vereinskorrespondenz, Fasz. 3 (1922-1940), Schreiben von Leodegar Petrin an die Landeskonservatorate, 11.8.1933. Ergänzt wird der Hinweis, dass „bedeutende Kunstwerke und Objekte von bedeutendem Denkmalwerte selbstverständlich nur erprobten Kräften anvertraut werden könnten“. 52 Beispielsweise kann hier auf den Abbruch des Gebäudes Reznicekgasse 20 (9. Bezirk) verwiesen werden. Siehe dazu: BDA-Archiv, Top. Mat., Wien IX. Bezirk, Profan, Reznicekgasse 20, 3881/Dsch/1937, Av Emmerich Siegris, 27.10.1937. 53 Ebd., Wien I. Bezirk, Profan, Singerstraße 30-32, 1382/Dsch/1935, Av Emmerich Siegris, 15.4.1935. 54 Einige verfügten über reizvolle Innenhöfe und wurden als Teil einer charakteristischen, ursprünglichen Bebauung erkannt oder bspw. als „für das Gesamtbild des [...] Stadtteiles von höchster Bedeutung“ kategorisiert. Siehe dazu: ebd., Wien I.Bezirk, Profan, Schönlaterngasse 4-7, 31/Dsch/1938, Av Karl Ginhart, 7.1.1938. $\mathbf{5 5}$ In der gesamten Zwischenkriegszeit wurden in ganz Österreich lediglich 250 Baudenkmale unter Schutz gestellt. Bernd Euler, Paul Mahringer, „Die Erhaltung der Architektur des 20. Jahrhunderts in Österreich. Routine und Neuland für Denkmalschutz und Denkmalpflege“, in: ÖZKD, 72. Jg., H. 3/4, 2018, S. 6-18, hier S. 7. 56 Bezugnehmend auf die von einer Privatperson eingeforderten Rettung der Lichtentaler Gasse (9. Bezirk) wird diese Auffassung durch eine Aktennotiz von Emmerich Siegris verdeutlicht: „Es ist natürlich unmöglich eine ganze Gasse unter Denkmalschutz zu stellen. Lichtental gibt sicher ein ganz gutes Bild des alten Wien. Kein Haus hat aber hohen Denkmalwert, doch wird dereinst um die trauten Winkel und Gassen sehr schade sein, wenn sie einmal weggerissen sind." Vgl. BDA-Archiv, Top. Mat., Wien IX. Bezirk, Profan, Lichtentalergasse, 4042/Dsch/1932, Av Emmerich Siegris, 7.9.1932. 57 Im Hinblick auf die fortgeschrittene Zerstörung des Stadtteils Lichtental bemerkt Referent Siegris: „Wenn auch, wie in Grinzing, das einzelne Haus unbedeutend erscheint, so ergeben sie in ihrer Anordnung und Gesamtheit doch ein reizvolles Ortsbild, das pietätvoll zu erhalten die Aufgabe des Wiener Stadtbauamtes wäre. Gegenwärtig ist man aber noch nicht so weit, denn es wird eine ganze Hauszeile angekauft und [...] niedergelegt." Vgl. ebd., Reznicekgasse 20, 3881/ Dsch/1937, Av Emmerich Siegris, 27.10.1937. 58 WStLA, 1.5.4. Stadtbaudirektion, A1, Allg. Reg., Schachtel 109/1937, GZ 4587/1937, Brief Stadtbaudirektor Musil an den Magistratsdirektor, 29.11.1937. 59 So etwa im Falle der Objekte Billrothstraße 51 (19. Bezirk) oder Windmühlgasse 28 (6. Bezirk), zu dem Referent Emmerich Siegris am 4.3.1936 vermerkte, dass eine Unterschutzstellung des „Geylinghauses“ die Zentralstelle gar „zu sehr belasten“ würde. Siehe dazu: BDA-Archiv, Top. Mat., Wien VI. Bezirk, Profan, Windmühlgasse 28, 637/Dsch/1936. 60 Ebd., Gumpendorferstraße 78, 1589/Dsch/1934, Av Emmerich Siegris, 29.11.1934. 61 BDA-Archiv, Top. Mat., Wien XIX. Bezirk, Profan 1, Cobenzlgasse 38 und 40, 1007/Dsch/1936, Av Emmerich Siegris, 7.4.1936. 62 Ebd., Kahlenbergerstraße 7-9, 2756/Dsch/1933, Av Emmerich Siegris, 21.7.1933. Siegris hält im Zusammenhang mit dem geplanten Abriss des Objektes Reznicekgasse 20 (9. Bezirk) mit emotionalem Unterton fest, dass „der Zerstörer" die Gemeinde Wien sei, die das Haus nun erworben habe. Ebd., Reznicekgasse 20,613/Dsch/1938, Av Emmerich Siegris, 23. 2.1938. 63 BDA-Archiv, Top. Mat., Wien XIX. Bezirk, Profan, Billrothstraße 60-62, 1220/Dsch/1936, Av Emmerich Siegris, 15.4.1936. 64 So Otto Demus im Jahr 1937 in Bezug auf den Teilabriss des Dominikanerklosters. Vgl. BDA-Archiv, Top. Mat., Wien Allg. 2, 1930-1945, div. Denkmäler bzw. Denkmalprojekte, 1730/Dsch/1937, Av Otto Demus, undatiert. 65 BDA-Archiv, Top. Mat., Wien I. Bezirk, Profan, Wollzeile 32, 3628/Dsch/1937, Antwortschreiben der Zentralstelle an den Oberstadtbaurat, 28.10.1937. 66 Ebd. 67 Ebd. 68 Im April 1928 schlug Referent Emil Ladewig vor, dass es „richtig wäre einmal und zwar bald eine Besprechung über die Regulierungsfragen zu halten, bei der auch die maßgebenden Faktoren der Gemeinde Wien“ zugegen sein sollten. Vgl. BDAArchiv, Top. Mat., Wien VI. Bezirk, Profan, Ratzenstadel, 2024/Dsch/1928, Av Referent Emil Ladewig, 19.4.1928. 69 Ginhart 1936, S.304. 70 BDA-Archiv, Top. Mat., Karton Wien Allg. 2, 1930-1945, div. Denkmäler bzw. Denkmalprojekte, 3574/Dsch/1937, Av Herbert Seiberl, undatiert, verm. nach dem 20.9.1937. 71 WStLA, 1.5.4. Stadtbaudirektion, A1, Allg. Reg., Schachtel 109/1937, GZ 4353/1937, Brief Clemens Holzmeister an Bgm. Schmitz, 26.10.1937. 72 Karl Oettinger, „Altes und Neues in der Stadtplanung Wiens", in: Wiener Zeitung, 2.3.1938, S. 7. Auch Hans Sedlmayr verweist auf die noch fehlende Wertschätzung des Barock bis 1895. Siehe dazu: ders., „Die Gefahr für Alt-Wien“, in: Neues Wiener Tagblatt, 6.3.1938, S. 9. 73 WStLA, 1.5.4. Stadtbaudirektion, A1, Allg. Reg., Schachtel 109/1937, GZ 4587/1937, Schreiben Stadtbaudirektor Franz Musil an den Magistratsdirektor, 
29.11.1937. 74 Ebd., Schreiben Stadtbaudirektor Franz Musil an Bgm. Schmitz, 22.11.1937. 75 Auch Emmerich Siegris hält fest: „Man will jetzt sogar so weit gehen, auch Wiens Vororte zu erhalten und der Zeitungstitel ,Denkmalschutz für Wiens Vororte' ist jedenfalls bezeichnend für den geistigen Umschwung im erstarrten Regulierungsbüro des Wiener Stadtbauamtes." Vgl. BDA-Archiv, Top. Mat., Wien XIX. Bezirk, Profan 1, Alt-Grinzing, 4413/ Dsch/1937, Av Emmerich Siegris, 16.12.1937. 76 Karl Oettinger, Assistent von Hans Sedlmayr am Institut für Kunstgeschichte der Universität Wien, verweist im Jänner 1938 auf die nicht durchgeführten Pläne für Grinzing und die Griechengasse, die in Kap. 4.7. noch genauer besprochen werden. Karl Oettinger, „Die Zerstörung der Singerstraße überflüssig“, in: Neues Wiener Tagblatt, 30.1.1938, S. 8. 77 „Wenn der Herr Bürgermeister jetzt den Wert unseres alten Stadtkernes zu würdigen beginnt, so ist das sehr erfreulich und noch nicht zu spät." BDA-Archiv, Top. Mat., Wien I. Bezirk, Profan, Griechengasse u. Hafnersteig, 255/Dsch/1938, Av Emmerich Siegris, 20.1.1938. 78 BDA-Archiv, Top. Mat., Wien Allg. 2, 1930-1945, div. Denkmäler bzw. Denkmalprojekte, 290/Dsch/1938, Brief Karl Giannoni an die Zentralstelle und Memorandum, 26.1.1938. 79 Ebd., 3189/Dsch/1938 und 4315/Dsch/ 1938. Zu diesem Zeitpunkt war Leodegar Petrin bereits zwangspensioniert, seine Nachfolge hatte Herbert Seiberl angetreten. 80 Oettinger, 2.3.1938, S.7. 81 BDA-Archiv, Top. Mat., Wien Allg. 2, 1930-1945, div. Denkmäler bzw. Denkmalprojekte, 3441/Dsch/1938, Abschrift des Antwortbriefes von Herbert Seiberl auf die Terminanfrage der Stadtbaudirektion, 7.10.1938. 82 BDA-Archiv, Top. Mat. Wien Allg. 2, 1930-1945, div. Denkmäler bzw. Denkmalprojekte, 4797/Dsch/1938, Brief Herbert Seiberl an Friedrich Otto, 5.1.1939. 83 Vgl. bspw.: BDA-Archiv, Top. Mat., Wien VI. Bezirk, Profan, 293/Dsch/1936, Kommentar des Referenten Emmerich Siegris zum Objekt Gumpendorfer Straße 89, 29.1.1936; ebd., 277/Dsch/1936, Kommentar des Referenten Emmerich Siegris zum Althaus in der Gumpendorfer Straße 134-136, 26.1.1936. 84 Alois Riegl, Der moderne Denkmalkultus. Sein Wesen, seine Entstehung, Wien/Leipzig 1903, S. 22-29. 85 Ebd., S. 16. 86 Karl Giannoni, „Entwickelung und Ziele des Heimatschutzes in Österreich", in: Gemeinsame Tagung für Denkmalpflege und Heimatschutz. Salzburg 14. und 15. September 1911, stenographischer Bericht, Berlin 1911a, S. 83-97, hier S. 84. 87 BDA-Archiv, Top. Mat., Wien I. Bezirk, Profan, Schönlaterngasse 4-7, 4061/Dsch/1937. 88 Ebd., 31/Dsch/1938, Rohfassung des Unterschutzstellungsbescheids, Karl Ginhart, Jänner 1938. 89 Ebd., 4061/Dsch/1937, Av Karl Ginhart, November 1937. 90 Siehe u. a.: Bundesdenkmalamt 1990, S. 146. 91 Als Beispiel sei hier, stellvertretend für viele andere, auf die Gebäudegruppe Strozzigasse 12-20 (8. Bezirk) verwiesen. Die fünf schmalen, einstöckigen Häuser mit „reizvollen Höfchen wurden als „künstlerisch [...] wertlos“ eingestuft. BDA-Archiv, Top. Mat., Wien VIII. Bezirk, Profan, Strozzigasse 16, 1043/ Dsch/1934. 92 Frodl-Kraft 1997, S. 83-84. 93 Dagobert Frey, „Tag für Denkmalpflege Würzburg und Nürnberg“, in: Zeitschrift für Denkmalpflege, 3. Jg., H. 1/2, 1928, S. 56-60, hier S.56. 94 Im Jahr 1930 wurde bspw. der Abbruch des Gebäudes Spittelberggasse 20 noch bewilligt, einige
Jahre später erteilte man für die bevorstehende Restaurierung der „künstlerisch bemerkenswerten Barockfassade" diverse Auflagen und Vorschriften. BDA-Archiv, Top. Mat., Wien VII. Bezirk, Profan, Spittelberggasse 20, 4004/ Dsch/1935. 95 So wurden etwa die Wandmalereien im Kreuzgang des zum Umbau bestimmten Dominikanerklosters als „belanglose Ornamente aus der Barockzeit“, als künstlerisch unbedeutend bewertet. BDA-Archiv, Top. Mat., Wien I. Bezirk, Profan, Dominikanerbastei, 2061/Dsch/1936, Mitteilung an den Prior, 6. 7.1936. 96 Zur Barockarchitektur in Österreich erschienen in den 1910er- und 1920erJahren vereinzelt Studien, in den frühen 1930er-Jahren: Hans Riehl, Barocke Baukunst in Österreich. Die Einführung des Barockstils in die deutsche Kunst (Die Kunst dem Volke, 73/74), München 1930; Hans Sedlmayr, Österreichische Barockarchitektur, 1690-1740, Wien 1930; Karl Ginhart (Hrsg.), Die bildende Kunst in Österreich. Barock und Rokoko, Baden bei Wien 1939. 97 So wurde die historistische Villa in der Himmelstraße 49 (19. Bezirk) als „wertlos" bezeichnet: BDA-Archiv, Top. Mat., Wien XIX.Bezirk, Profan, Himmelstraße 49, 840/Dsch/1936, Av Emmerich Siegris, 13.3.1936; und als „belangloses Objekt aus den 90er Jahren des vorigen Jahrhunderts": ebd., 1044/Dsch/1936, Av Emmerich Siegris, 28.3.1936. 98 Hinsichtlich der Niveauänderung um die Staatsoper, „die sich sehr empfindlich bemerkbar und dem schönen Baue merklich Abbruch in seiner Wirkung getan hätte“, konnte die Zentralstelle nur durch ein „energisches Einschreiten“ Schlimmeres verhindern. Siehe dazu: Ginhart 1936, S. 304. 99 Bspw. war das Gebäude Beatrixgasse 5-7 „ein reizvolles Biedermeierhaus aus der Zeit des Wiener Vormärz wie sie noch sehr zahlreich vorhanden sind“, nicht erhaltenswert. BDA-Archiv, Top. Mat., Wien III. Bezirk, Profan, Beatrixgasse 5-7, 1044/Dsch/1934, Av Emmerich Siegris, 20.10.1934. 100 Es wurde wiederum lediglich eine Fotodokumentation eingefordert. BDA-Archiv, Top. Mat., Wien IX.Bezirk, Profan, Althangasse 23, 6617/Dsch/1928, Av Emmerich Siegris, 28.12.1928. 101 Ebd., Marktgasse 26, 592/Dsch/1929, Av Emmerich Siegris, 29.1.1929. 102 Vgl. Emmerich Siegris' Kommentar zur Hofmühlgasse 20 vom 5.11.1934: „Biedermeierhäuser haben stimmungsvolle Höfe aber keinen Kunstwert.“ BDA-Archiv, Top. Mat., Wien VI. Bezirk, Profan, Hofmühlgasse 20, 1274/Dsch/1934. 103 Petrin 1937, S. 60. 104 Es sei hier auf die Beschreibungen der Assanierungsbauten in Kap. 3 verwiesen. Zahlreiche Skulpturen wurden in die Fassaden der Neubauten integriert, bspw. im Falle des Neubaus Singerstraße 30-32. 105 So schlug Referent Siegris 1937 vor, den Fassadenschmuck des ehemaligen Hauptpostamts (Fleischmarkt 19) „an dem neuen Gebäude anzubringen, um inn auch für die Zukunft erhalten zu können“. Siehe dazu: BDA-Archiv, Top. Mat., Wien I. Bezirk, Profan, Fleischmarkt 19, 695/Dsch/1937, Av Emmerich Siegris, 16. 2.1937. 106 So etwa bei der Bauverhandlung zum geplanten Abriss des Objektes Lerchenfelder Straße 50, hier sollte die Statue des HI. Antonius in die Fassade des Neubaus integriert werden: BDA-Archiv, Top. Mat., Wien VIII. Bezirk, Profan, Lerchenfelderstraße 50, 1069/Dsch/ 1936, Av Emmerich Siegris, 1.5.1936. Beim Umbau des Dominikanerklosters in der Inneren Stadt plädierte die Zentralstelle für die Erhaltung des sogenannten 
„Windhag'schen Portals“: BDA-Archiv, Top. Mat., Wien I. Bezirk, Profan, Dominikanerbastei, 3425/Dsch/1936, Av Emmerich Siegris, 10.11.1936. 107 Ebd., Wollzeile 30, 96/Dsch/1938, Gesprächsprotokoll, 11.1.1938. 108 Bspw. wurde das Objekt Nussgasse 3-5 (9. Bezirk) aus diesem Grund als nicht schutzwürdig befunden: „Die Häuser haben nur lokalhistorischen Wert und müssen wegen Baufälligkeit demoliert werden. Denkmalschutz kommt nicht in Frage." BDA-Archiv, Top. Mat., Wien IX. Bezirk, Profan, Nussgasse 3-5, 58/Dsch/1932, Av Emmerich Siegris, 6.1.1932. 1091934 hatte die Zentralstelle gegen den bevorstehenden Abriss noch Einspruch erhoben und den Antrag des Assanierungsfonds abgelehnt. Der Abbruch des „reizvollen Barockschlösschens“ hätte einen „unersetzlichen [nachträglich gestrichen, Anm. d. Autorin] Verlust in künstlerischer Beziehung" bedeutet. BDA-Archiv, Top. Mat., Wien VI. Bezirk, Profan, Windmühlgasse 28, 1518/ Dsch/1934, Av Emmerich Siegris, undatiert. 110 Ebd., 637/Dsch/1936, Abschrift eines Antwortbriefes von Emmerich Siegris, 4.3.1936. 111 BDA-Archiv, Top. Mat., Wien XIX. Bezirk, Profan 1, Alt-Grinzing Ortsbild, 2406/ Dsch/ 1937, Brief des Stadtbauamtes an die Zentralstelle, 2.7. 1937. 112 Vgl. dazu div. Zeitungsartikel, bspw.: ebd., 4413/Dsch/1937, „Denkmalschutz für Wiens Vororte. Alle historischen Bauten bleiben erhalten“, in: Wiener Journal, 16.12.1937. $113 \mathrm{Vgl}$. dazu diverse Zeitungsberichte, bspw.: ebd., 4049/Dsch/1937, „Vor der Entscheidung im Grinzinger Wettbewerb“, in: Reichspost, 9.11.1937, S. 5.
114 Ebd., 2406/Dsch/1937, Antwortschreiben Präsident Petrin an die Stadtbaudirektion, 7.7.1937. 115 „Zum Grinzinger Wettbewerb“, in: Österreichische Bauzeitung, 3. Jg., Folge 6, 15.2.1938, S.65-71, hier S.65. 116 Emmerich Siegris: „Der alte Weinbauort Grinzing und die Bestrebungen, sein Ortsbild zu erhalten“, in: Jahrbuch für Landeskunde von Niederösterreich, Bd. 27, 1938, S. 294-302, hier S. 302. Dennoch wurden während des Wettbewerbs in Grinzing Demolierungsarbeiten vorgenommen. Siehe dazu: „Ist Alt-Grinzing bereits verloren?“, in: NeuigkeitsWelt-Blatt, 14.8.1937, S. 4. 117 K. K. Zentral-Kommission für Kunst- und Historische Denkmale, 1916, S. 60. 118 Ebd., S. 57. 119 BDA-Archiv, Top. Mat., Wien I. Bezirk, Profan, Griechengasse u. Hafnersteig, 591/Dsch/1938. 120 Die beiden betroffenen Gebäude Griechengasse 2 und 4 werden im „Kunsthistorischen Plan“ von Hugo Hassinger auf das frühe 19. Jh. datiert, über die im Inneren und im Bereich der Fassade von Haus Nr. 4 erhaltenen deutlich älteren Teile war damals offensichtlich noch nichts bekannt; eine wesentliche Verbreiterung sah der geänderte Regulierungsplan allerdings auch im unteren Bereich der Griechengasse vor. 121 BDA-Archiv, Top. Mat., Wien I. Bezirk, Profan, Griechengasse u. Hafnersteig, 255/Dsch/1938, Av Emmerich Siegris, 20.1.1938. 122 Ebd., Dorotheergasse 11, 3677/Dsch/1935, Av Emmerich Siegris, 7.11.1935. 123 Ebd., Stoß im Himmel 3, 3626/Dsch/1935, Av Emmerich Siegris, 21.10.1935. 


Kapitel 5 